\title{
Molecular switches regulating the potency and immune evasiveness of SARS-CoV-2 spike protein
}

\author{
Yushun Wan \\ University of Minnesota \\ Linfen Huang \\ University of Minnesota
}

Xiujuan Zhang

Lindsley F. Kimball Research Institute

Jian Shang

University of Minnesota

Stanley Perlman

University of lowa

Lanying Du

Lindsley F. Kimball Research Institute

Fang Li ( $\sim$ lifang@umn.edu )

University of Minnesota

\section{Research Article}

Keywords: SARS-CoV-2, spike protein, receptor-binding domain, ACE2 binding, viral entry, immune evasiveness, cryo-EM structures

Posted Date: October 1st, 2021

DOI: https://doi.org/10.21203/rs.3.rs-736159/v2

License: (c) (i) This work is licensed under a Creative Commons Attribution 4.0 International License.

Read Full License 


\section{Molecular switches regulating the potency and immune evasiveness} of SARS-CoV-2 spike protein

$5 \quad$ Yushun Wan ${ }^{1,2}$, Linfen Huang ${ }^{1,2}$, Xiujuan Zhang ${ }^{3}$, Jian Shang ${ }^{1,2}$,

$8{ }^{1}$ Department of Veterinary and Biomedical Sciences, University of Minnesota, Saint

9 Paul, MN, USA

$10{ }^{2}$ Center for Coronavirus Research, University of Minnesota, Saint Paul, MN, USA

$11{ }^{3}$ Laboratory of Viral Immunology, Lindsley F. Kimball Research Institute, New York

12 Blood Center, New York, NY, USA

$13{ }^{4}$ Department of Microbiology and Immunology, University of Iowa, Iowa City, IA, USA

16 \# Correspondence:

17

18 Fang Li (lifang@umn.edu)

19

20

21 Keywords: SARS-CoV-2, spike protein, receptor-binding domain, ACE2 binding, viral

22 entry, immune evasiveness, cryo-EM structures

23

24 Running title: Dynamics between potency and evasiveness of SARS-CoV-2 


\section{Abstract}

27 SARS-CoV-2 spike protein plays a key role in viral entry and host immune

28 responses. The conformation of the spike protein can be either open or closed, yet it is

29 unclear how the conformations affect the protein's functions or what regulate the

30 conformational changes. Using SARS-CoV-1 and bat RaTG13-CoV as comparisons, we

31 identified two molecular switches that regulate the conformations of SARS-CoV-2 spike

32 protein: (i) a furin motif loop turns SARS-CoV-2 spike from a closed conformation to a

33 mixture of open and closed conformations, and (ii) a K417V mutation turns SARS-CoV-

342 spike from mixed conformations to an open conformation. We showed that the open

35 conformation favors viral potency by exposing the RBD for receptor binding and viral

36 entry, whereas the closed conformation supports viral immune evasion by hiding the

37 RBD from neutralizing antibodies. Hence SARS-CoV-2 spike has evolved to reach a

38 balance between potency and immune evasiveness, which may contribute to the

39 pandemic spread of SARS-CoV-2. The dynamics between viral potency and invasiveness

40 is likely to further evolve, providing insights into future evolution of SARS-CoV-2. 


\section{Introduction}

44 Coronaviruses have a long history of infecting humans and animals, yet none had 45 caused the same devastation as produced by $\operatorname{SARS}-\mathrm{CoV}-2(1,2)$. For example, a virulent 46 and lethal coronavirus, SARS-CoV-1, yielded a much smaller outbreak in humans in

47 2002-2003 (3, 4). Numerous human coronaviruses such as NL63-CoV cause common

48 colds annually $(5,6)$. With an intermediate virulence, SARS-CoV-2 causes a fatality rate

49 that is significantly lower than that of SARS-CoV-1, but much higher than that of NL63-

50 CoV. SARS-CoV-2 carriers show clinical signs that facilitate the spread of the virus: they

51 may develop mild or no symptoms, experience delayed onset of symptoms, develop low

52 levels of neutralizing antibodies, or endure prolonged virus shedding period (7-11). These

53 features contribute to the wide spread of SARS-CoV-2 and severe health outcomes,

54 triggering a global COVID-19 pandemic that is unprecedented in the era of modern

55 medicine. Understanding the molecular determinants of COVID-19 provides important

56 clues to the evolution and cross-species transmission of coronaviruses. A dangerous

57 feature of coronaviruses is their propensity to cross species barriers $(12,13)$. In fact,

58 coronaviruses similar to human coronaviruses such as SARS-CoV-1 and NL63-CoV have

59 been identified in bats and other animals (14-16). RaTG13-CoV, a coronavirus with

$60 \sim 96 \%$ genomic sequence homology with SARS-CoV-2, has been identified in bats (17).

61 Thus, coronaviruses that originate from bats or other animals pose a long-term threat to

62 humans. A comparison of the molecular mechanisms of SARS-CoV-2 and other

63 coronaviruses not only facilitate an understanding of the COVID-19 pandemic, but also

64 shed light on the evolution of coronaviruses, including their cross-species transmission

65 and adaptation to humans. 
The viral-envelope-anchored spike protein guides coronavirus entry into host cells

67 (18). At the same time, it is a major target for the host immune responses (19). On newly

68 packaged virus particles, the trimeric spike protein has a pre-fusion structure in which

69 three receptor-binding S1 subunits sit on top of a trimeric membrane-fusion S2 stalk (Fig.

70 1A, 1B). During viral entry, a receptor-binding domain (RBD) in S1 binds to a receptor

71 on host cell surface for viral attachment (20); subsequently S1 dissociates and S2

72 switches to a post-fusion structure for the fusion of viral and host membranes (18). For

73 the pre- to post-fusion structural change to take place, all coronavirus spikes need to be

74 cleaved by host proteases $(21,22)$. SARS-CoV-2, SARS-CoV-1, NL63-CoV and

75 RaTG13-CoV can all use ACE2 as the receptor (17, 23-26), but SARS-CoV-2 spike

76 contains two unique features. First, only SARS-CoV-2 spike contains a furin motif at the

77 S1/S2 boundary (27), which allows SARS-CoV-2 spike to be pre-activated by furin from

78 previously infected cells. Second, the pre-fusion structure of SARS-CoV-2 is present in

79 two conformations with approximately equal ratio: an open conformation in which the

80 RBD is exposed and accessible to ACE2 and a closed conformation in which the RBD is

81 buried and inaccessible to ACE2 $(28,29)$. In contrast, SARS-CoV-1 spike is mainly open

82 and NL63-CoV and RaTG13-CoV spikes are only closed (30-32). Yet despite the

83 extensive structural studies of coronavirus spikes (Table S1), it is unclear what molecular

84 switches regulate their conformations or how the conformational changes affect viral

85 functions and host immune responses.

86 Here we compared the spike proteins of the three ACE2-recognizing

87 coronaviruses. Using biochemical, pseudovirus, cryo-EM, and animal immunization

88 assays, we first identified the molecular switches that regulate the RBD conformations in 
89 SARS-CoV-2 spike. We then demonstrated that whereas the open conformation of

90 SARS-CoV-2 spike increases its potency, the closed conformation allows it to evade host

91 immune responses. Through regulation of its spike's conformations, SARS-CoV-2 may

92 have struck a balance between viral potency and evasiveness. The dynamics of this

93 balance may further evolve, shedding light on future evolution of SARS-CoV-2.

95 Results

96 To understand the molecular mechanisms that control the spike RBD switching

97 between open and closed conformations, we conducted a comparative study of SARS-

$98 \mathrm{CoV}-2$ and the other ACE2-recognizing coronaviruses. Sequence analysis showed that

99 compared to the closely related SARS-CoV-1 and RaTG13-CoV, only SARS-CoV-2

100 spike contains a four-residue PRRA insertion ahead of a conserved Arg685 at the S1/S2

101 junction, constituting the furin motif (FnM) (Fig. 1A, 1B). Hence we introduced

102 mutations to inactivate the FnM in SARS-CoV-2 spike in three ways: (i) point mutations

103 from PRRA to PAGA (i.e., FnM-point); (ii) deletion mutation based on SARS-CoV-1

104 spike (i.e., FnM-deletion); (iii) deletion mutation based on RaTG13-CoV (i.e., FnM-

105 deletion-2). We then explored whether these FnM mutations affected the conformation

106 and potency of SARS-CoV-2 spike.

107 To this end, we characterized the capabilities of the FnM mutant spikes of SARS-

108 CoV-2 in binding human ACE2 and mediating viral entry. First, we expressed the wild

109 type and mutant spikes on cell surface (Fig. 1C). The result showed that during the

110 maturation process, a significant amount of wild type spike molecules had been cleaved

111 by furin. In contrast, none of the three types of FnM mutant spikes had undergone 
112 significant cleavage, suggesting that inactivation of FnM successfully suppressed furin

113 cleavage of the spikes. Second, we performed a protein pull-down assay using

114 recombinant human ACE2 as the bait and the cell-surface-anchored spikes as the target.

115 For cross validation, both His-tagged ACE2 and Fc-tagged ACE2 were used. We

116 previously showed that this pull-down assay is a reliable method to probe the RBD

117 conformation in cell-surface-anchored spikes, with higher pull-down levels of the spikes

118 associated with more spike molecules in the RBD-open conformation (27). Our results

119 showed that the wild type and FnM-point spikes had similar affinities for ACE2, and both

120 demonstrated much higher affinities for ACE2 than the two FnM-deletion spikes (Fig.

121 1C). Third, we performed a pseudovirus entry assay where retroviruses pseudotyped with

122 SARS-CoV-2 spike (i.e., SARS-CoV-2 pseudoviruses) were used to enter cells

123 expressing human ACE2 (Fig. 1D). The result showed that the FnM-point spikes

124 mediated pseudovirus entry slightly worse than the wild type spike, suggesting that furin

125 pre-activation had small, albeit significant, impact on SARS-CoV-2 spike's capability in

126 mediating viral entry. In contrast, both of the FnM-deletion spikes mediated pseudovirus

127 entry much worse than both the wild type spike and FnM-point spike, suggesting that the

128 closed conformation of the spike substantially reduced its capability to mediate viral

129 entry. The data from protein pull-down and pseudovirus entry assays revealed that FnM

130 deletion resulted in decreased potency of SARS-CoV-2 spike, as demonstrated in reduced

131 ACE2 binding and reduced capability of mediating viral entry. These results suggest that

132 due to the FnM deletion, more SARS-CoV-2 spike molecules switched to the closed

133 conformation with reduced potency. 
135 the FnM deletion using cryo-EM. To this end, we expressed and purified the ectodomain

136 of SARS-CoV-2 spike containing the FnM deletion (it also contained a C-terminal foldon

137 trimerization tag and two proline mutations in S2, both of which stabilize the pre-fusion

138 structure). As a comparison, we also prepared the ectodomain of SARS-CoV-2 spike

139 containing the FnM point mutation (in addition to the foldon tag and proline mutations).

140 We then collected cryo-EM data on both of these proteins and performed 3-D

141 classifications of the particles based on their conformations (Fig. 2A, 2B; Fig. S1). Our

142 results showed that $48 \%$ of FnM-point spike molecules are in the open conformation with

143 one of the three RBDs exposed and the $52 \%$ of the molecules were in the closed

144 conformation with all three RBDs hidden (Fig. 2B). This result is consistent with two

145 previous studies showing an approximately equal ratio of open and closed spike

146 molecules (one of the studies involved recombinant FnM-point spike ectodomain and the

147 other virus-surface wild type full-length spike) $(28,29)$. In contrast, our cryo-EM result

148 showed that all of the FnM-deletion spike molecules were in the closed conformation

149 with all three RBDs hidden (Fig. 2A). Therefore, consistent with our biochemical data,

150 our cryo-EM data confirmed that the FnM deletion caused SARS-CoV-2 spike to switch

151 to the closed conformation.

152 We further determined the cryo-EM structures of SARS-CoV-2 FnM-deletion

153 spike ectodomain at $3.8 \AA$ and FnM-point spike ectodomain at $4.4 \AA$ (Fig. 2A, 2B; Fig.

154 S2A, Fig. S2B). Overall, the two structures are similar to each other and to the previously

155 determined cryo-EM structures of FnM-point spike ectodomain and virus-surface wild

156 type full-length spike $(28,29)$. In the trimeric spike structures, each S1 subunit contains 
157 an N-terminal domain (NTD), an RBD, and two subdomains (SD1 and SD2); the RBD

158 from one S1 subunit packs against the NTD from another S1 subunit and it also packs

159 against the two RBDs from the other two S1 subunits (Fig. S3A) (28). Moreover, the

160 RBD switches between the open and closed conformations by rotating around a hinge

161 region connecting SD1 and SD2; SD2, which harbors the FnM loop, directly interacts

162 with the hinge region and the NTD (Fig. S3A) (28). Detailed structural analysis revealed

163 that compared to the FnM-point spike, the RBD and NTD in each S1 subunit of the FnM-

164 deletion spike rotated towards each other by $\sim 2.5^{\circ}$ (Fig. S4A). Because of this movement,

165 compared to the FnM-point spike, the RBD/NTD interface, the RBD/RBD interface and

166 hence the total interface in trimeric S1 all increased significantly in the FnM-deletion

167 spike, leading to enhanced S1 packing (Fig. S3B). As a comparison, the corresponding

168 interfaces in a previously determined FnM-point spike were similar to those in our FnM-

169 point spike (Fig. S3B) (28). What caused this structural change is not obvious due to the

170 lack of density in the FnM loop and another loop (i.e., anchor loop) in SD2 from all the

171 available structures of SARS-CoV-2 spike. However, the structures of the FnM loop and

172 the anchor loop were resolved in the mouse hepatitis coronavirus (MHV) spike structure

173 that we recently determined (33). Because the MHV and SARS-CoV-2 spikes have

174 overall similar structures (Fig. S4B), we combined the structural information from these

175 two spikes, which revealed an interaction network involving the FnM loop, anchor loop,

176 the hinge region, and the NTD (Fig. S4B). Hence, one possibility is that the FnM deletion

177 disturbed this interaction network and caused the movements of the RBD and NTD,

178 which subsequently led to enhanced S1 packing, reduced dynamics of the RBD and

179 hence the closed spike. Thus, as supported by the biochemical data and 3D classification 
180 data, the physical presence of the FnM, instead of furin cleavage per se, leads to open

181 spike molecules by reducing S1 packing.

182 To further understand the relationship between the presence of FnM and the

183 conformation of the spikes, we inserted FnM into RaTG13-CoV spike (i.e., FnM-insert)

184 (Fig. 3A). As a comparison, we also inserted a random sequence, glycine-serine-glycine-

185 serine, into the same location as the inserted FnM in RaTG13-CoV spike (i.e., GSGS-

186 insert) (Fig. 3A). When expressed on cell surfaces, FnM-insert spike, but not wild type

187 spike or GSGS-insert spike, was cleaved by furin (Fig. 3B), confirming the introduction

188 of FnM. We could not obtain recombinant RaTG13 spike ectodomains (wild type or

189 mutants) that were stable enough for cryo-EM analysis (recombinant spike ectodomains

190 are generally less stable than full-length membrane-anchored spikes). Instead, we

191 examined the RBD conformations of the mutant spikes using protein pull-down and

192 pseudovirus entry assays (Fig. 3B, 3C). Compared to the wild type spike, both the FnM-

193 insert and FnM-GSGS RaTG13-CoV spikes bound to human ACE2 with higher affinity

194 and mediated pseudovirus entry more efficiently. Thus, the physical presence of FnM or

195 another random sequence in the FnM loop opens up RaTG13-CoV spike and enhances its 196 potency.

197 Having identified the FnM loop as a key determinant for the conformation of

198 SARS-CoV-2 spike, we asked why SARS-CoV-1 spike is in the open conformation

199 despite its lack of FnM. To address this question, we compared the sequences of SARS-

200 CoV-2 and SARS-CoV-1 spikes in the context of their tertiary structures. We identified

201 residue 417 as potentially a key difference between the two spikes: in the closed SARS-

202 CoV-2 spike, Lys417 in the RBD forms a salt bridge with the RBD from another subunit, 
203 stabilizing the RBD in the closed conformation and hence enhancing S1 packing; it

204 becomes a valine in SARS-CoV-1 spike, losing its capability to interact with the other

205 subunit and hence reducing S1 packing (Fig. 4A). We introduced the K417V mutation

206 into SARS-CoV-2 spike, and examined its impact on the conformation of SARS-CoV-2

207 spike. Both the protein pull-down and pseudovirus entry assays demonstrated that

208 compared to the wild type spike, the K417V mutation allowed more spike molecules to

209 open up for binding ACE2 and mediating viral entry (Fig. 4B, 4C). We could not obtain

210 recombinant SARS-CoV-2 K417V spike ectodomain that was stable enough for cryo-EM

211 analysis. Instead, we prepared recombinant SARS-CoV-2 spike ectodomain containing

212 the K417V mutation and FnM deletion (in addition to proline mutations) (K417V/FnM-

213 deletion). Cryo-EM analysis at $4.6 \AA$ revealed that $91 \%$ of the $\mathrm{K} 417 \mathrm{~V} / \mathrm{FnM}$-deletion

214 spike molecules were open and 9\% were closed (Fig. 2C). In comparison, as presented

215 earlier, 100\% of the recombinant FnM-deletion spike molecules were closed (Fig. 2A).

216 Therefore, despite lacking FnM, SARS-CoV-1 spike is open due to Val417 and

217 potentially other residues that destabilize the closed conformation of the RBD and reduce

218 S1 packing.

219 To understand how the RBD conformations of SARS-CoV-2 spike affect host

220 immune responses targeting the RBD, we immunized mice with one of the following

221 three recombinant SARS-CoV-2 spike ectodomains: FnM-deletion spike, FnM-point

222 spike, and $\mathrm{K} 417 \mathrm{~V} / \mathrm{FnM}$-deletion spike (in addition to the proline mutations in all of

223 them). Four weeks after the initial immunization, the mice were further boosted with the

224 same immunogen. Ten days after the second immunization, mouse sera were collected.

225 We measured the amounts of RBD-specific antibodies in the mouse sera using ELISA. 
226 The result showed that K417V/FnM-deletion spike and FnM-point spike induced

227 significantly more RBD-specific antibodies than FnM-deletion spike (Fig. 5A). We

228 further measured the amounts of neutralizing antibodies in the mouse sera using

229 pseudovirus entry inhibition assay. The result showed that K417V/FnM-deletion spike

230 and FnM-point spike induced significantly more neutralizing antibodies than FnM-

231 deletion spike (Fig. 5B). These data confirm that more molecules of K417V/FnM-

232 deletion spike and of FnM-point spike are in the open conformations than FnM-deletion

233 spike. They also reveal that compared to open spikes, closed spikes trigger lower levels

234 of RBD-targeting antibodies and neutralizing antibodies and hence their RBDs and spikes

235 are more evasive to the host immune system.

236 To summarize, we investigated the molecular switches regulating the

237 conformation of SARS-CoV-2 spike protein. We used four different experimental

238 approaches: pull-down of cell-surface spikes, cryo-EM analysis of recombinant spike

239 ectodomains, spike-mediated pseudovirus entry, and immunization of mice with

240 recombinant spike ectodomains. To date, several other studies also investigated the

241 conformations of SARS-CoV-2 spike using cryo-EM $(28,29,31,34,35)$, some of which

242 gave different ratios of open and closed spikes probably due to differences in sample

243 preparations and/or protein constructions. The ratio of open and closed spikes in our

244 FnM-point construct is similar to two other studies: a study that examined the full-length

245 virus-anchored SARS-CoV-2 spike (which is likely the most physiologically relevant)

246 (29) and a study that used the same protein construct and similar protein preparation to

247 the current study (28). Importantly, our cryo-EM analysis is consistent with our three

248 other experimental approaches. These different experimental approaches complement 
249 each other and make this study among the most comprehensive in investigating the

250 conformations of SARS-CoV-2 spike.

\section{Discussion}

253 Several molecular features of SARS-CoV-2 may have contributed to the COVID-

25419 pandemic. Among them are features involving SARS-CoV-2 spike: high ACE2-

255 binding affinity of the RBD, the presence of the furin motif at the S1/S2 boundary, and

256 the RBD switching between open and closed conformations (26-28). The molecular

257 mechanisms for the first two have been well established in previous research on SARS-

258 CoV-1 and MERS-CoV (MERS-CoV spike contains a furin motif) $(18,20,36)$. These

259 two features facilitated SARS-CoV-1 and MERS-CoV, respectively, to infect humans. In

260 this study we examined the molecular mechanisms that regulate the conformations of

261 SARS-CoV-2 spike. Our study showed that the conformations of coronavirus spike

262 proteins are regulated through one or a few molecular and structural switches. Our study

263 also clarified the functions of these conformational changes of coronavirus spikes. It has

264 implications for the structure, function and evolution of coronavirus spikes and for the

265 current and potentially future coronavirus infections.

266 How do the conformations of SARS-CoV-2 spike impact viral entry and host

267 immune responses? Among the coronavirus spikes whose tertiary structures are available,

268 only three showed a significant presence of open conformations in cryo-EM studies:

269 those from SARS-CoV-2, SARS-CoV-1, and MERS-CoV (Table S1). All three are novel

270 coronaviruses that recently infected humans. In contrast, coronaviruses with closed spikes

271 all have established infections in their respective hosts (the spikes would need to open 
272 briefly for receptor binding). This difference suggests that the open spike may facilitate

273 novel coronaviruses to infect humans. Consistent with this hypothesis, here we showed

274 that compared to closed spikes, open spikes mediate more efficient receptor binding and

275 viral entry. On the other hand, SARS-CoV-2 spike has a balanced ratio between open and

276 closed conformations, which may enhance immune evasion during its transmission in

277 humans. Consistent with this hypothesis, here we showed that more spike molecules in

278 the closed conformation correspond to decreased inductions of RBD-targeting antibodies

279 and neutralizing antibodies in mice. Together, these findings demonstrate that the

280 presence of open and closed conformations of its spike allows SARS-CoV-2 to balance

281 its potency and immune evasiveness.

282 How has SARS-CoV-2 spike evolved to reach the balance of potency and

283 evasiveness? Through comparative studies of the spikes from SARS-CoV-2, SARS-CoV-

2841 and RaTG13-CoV, we identified two molecular switches for the open and closed

285 conformational changes of SARS-CoV-2 spike: the physical presence of the furin motif

286 loop and the mutation of residue 417, both of which regulate S1 packing. Thus, one or

287 several structural changes in coronavirus spikes can function as molecular switches for

288 the conformations of coronavirus spikes. Other molecular determinants may also exist to

289 control the opening and closing of coronavirus spikes, but these two naturally occurring

290 molecular determinants help understand the evolution of coronavirus spikes.

291 Coronaviruses that have evolved molecular switches to open up their spike may have an

292 advantage in spreading efficiently in the infected host species. In contrast, those that have

293 evolved mechanisms to close down their spikes may have an advantage in establishing

294 evasive and long-lasting infections in the infected host species. 
These results further our understanding of the molecular mechanisms for the

296 COVID-19 pandemic. First, adaptation of SARS-CoV-2 RBD to human ACE2 and furin

297 cleavage of SARS-CoV-2 spike both play important roles in the transmission of SARS-

298 CoV-2 in humans. Second, a balanced open and closed RBD conformations of SARS-

299 CoV-2 spike enable the virus to be both potent and immune evasive. Unlike the stepwise

300 accumulation of point mutations in the RBD for enhanced ACE2 binding, the molecular

301 switches for the RBD conformations of the spike allow more drastic and efficient control

302 of ACE2 binding and viral entry. The opening up of its spike likely facilitates SARS-

303 CoV-2 to gain infection potency and spread efficiently in humans. Moreover, with

304 balanced conformations of its spike, SARS-CoV-2 is also immune evasive. This feature

305 of SARS-CoV-2 may contribute to the relatively long incubation time, harder-to-detect

306 symptoms (even asymptomatic infections), low neutralizing antibodies, or long virus

307 shedding period in patients; these clinical symptoms of patients may further contribute to

308 the wide spread of SARS-CoV-2. Therefore, the balanced potency and immune

309 evasiveness of SARS-CoV-2 spike may contribute to the wide spread of SARS-CoV-2.

310 Our findings also provide insights into how SARS-CoV-2 may further evolve.

311 When SARS-CoV-2 first entered humans, its spread met little immune resistance. The

312 more open and potent spike gave the virus an advantage in spreading widely. Several

313 months in the pandemic, a D614G mutation allowed more SARS-CoV-2 spike molecules

314 to open up (37,38), a sign that the virus was gaining more potency (39). Eventually,

315 however, as infection cases rise and vaccinations get underway, SARS-CoV-2 may

316 evolve towards better immune evasiveness. This may happen through an increase in the

317 proportion of closed spikes, making the virus more immune evasive but less potent. If 
318 that happens, SARS-CoV-2 may become an endemic (but milder) virus like NL63-CoV

319 (NL63-CoV RBD binds to human ACE2 with high affinity, but is hidden in the closed

320 spike) $(25,30,40)$. This study showed that just one or a few structural changes in the

321 spike protein can significantly impact the dynamics between viral potency and

322 evasiveness. This makes coronaviruses a current and future danger to human health.

323 Understanding the molecular determinants that regulate the potency and evasiveness of

324 coronaviruses is critical not only for our understanding the current COVID-19 pandemic,

325 but also for monitoring and preparing for potential future coronavirus pandemics. 


\section{Acknowledgements}

327 This work was supported by NIH grants R01AI089728 and R01AI110700 (to

328 F.L.) and NIH grants R01AI139092 (to F.L. and L.D.). Cryo-EM data were collected at

329 the John M. Cowley Center for High Resolution Electron Microscopy of Arizona State

330 University and the Electron Microscopy facility of Purdue University. We thank Dewight

331 Williams, Wei Zhang and Thomas Klose for helping us prepare grids and collect cryo-

332 EM data. The cryo-EM maps and atomic models have been deposited in the Electron

333 Microscopy Data Bank (EMDB) and Protein Data Bank (PDB), respectively, under the

334 following accession codes: EMD-9409 (EMDB) and 5BKP (PDB) for FnM-deletion

335 SARS-CoV-2 spike and EMD-9408 (EMDB) and 5BKO (PDB) for FnM-point SARS-

336 CoV-2 spike. We thank Professor Kathryn Holmes for discussion and edits to the

337 manuscript.

338 


\section{Materials and Methods}

340 Plasmids

341 All of the protein constructs in this study were cloned into pcDNA 3.1 vector

342 (Life Technologies). SARS-CoV-2 spike (GenBank accession number QHD43416.1),

343 SARS-CoV-1 spike (GenBank accession number YP_009825051.1), RaTG13-CoV spike

344 (GenBank accession number QHR63300.2), and human ACE2 (GenBank accession

345 number NM_021804) were all synthesized (GenScript Biotech) and cloned into the

346 vector containing a C-terminal c9 tag. SARS-CoV-2 spike ectodomains (residues 1-1211)

347 were cloned into the vector containing mutations of interest, in addition to two proline

348 mutations in S2 (K986P, V987P), a C-terminal foldon trimerization tag, and a C-terminal

349 His $_{6}$-tag. SARS-CoV-2 spike RBD (residues 319-535) and SARS-CoV-1 spike RBD

350 (residues 306-521) were cloned into the vector containing an N-terminal tPA signal

351 peptide. Human ACE2 ectodomain (residues 1-615) were cloned into the vector

352 containing either a C-terminal $\mathrm{His}_{6}$-tag or Fc-tag.

353 Protein expression and purification

$354 \quad$ All of the recombinant proteins were expressed in 293F cells (Thermo Fisher)

355 using a FreeStyle 293 mammalian cell expression system (Life Technologies) as

356 previously described (41). In brief, the His-tagged proteins were collected from cell

357 culture medium, purified using a Ni-NTA column (Cytiva Healthcare), purified further

358 using a Superdex gel filtration column (Cytiva Healthcare), and stored in a buffer

359 containing $20 \mathrm{mM}$ Tris $\mathrm{pH} 7.4$ and $200 \mathrm{mM} \mathrm{NaCl}$. The Fc-tagged protein was purified in

360 the same way as the His-tagged proteins, except that the protein A column replaced the

361 Ni-NTA column in the procedure. 
363 Pseudoviruses were packaged as previously described (42). Briefly, pcDNA3.1(+)

364 plasmid encoding one of the full-length coronavirus spike genes (wild type or mutant)

365 was co-transfected into HEK293T cells with helper plasmid psPAX2 and reporter

366 plasmid plenti-CMV-luc at a molar ratio of 1:1:1 using Lipofectamine 3000 (Life

367 Technologies). The produced pseudoviruses were harvested 72 hours post transfection

368 and then were used to enter HEK293T cells expressing human ACE2. After incubation at

$36937^{\circ} \mathrm{C}$ for 5 hours, medium was replaced and cells were incubated for an additional 48

370 hours. Cells were then washed with PBS and lysed. Aliquots of cell lysates were

371 transferred to Optiplate-96 (PerkinElmer), followed by addition of luciferase substrate.

372 Relative light unites (RLUs) were measured using EnSpire plate reader (PerkinElmer). In

373 the meanwhile, the amounts of pseudovirus-packaged spikes were measured by western

374 blot using an anti-c9 antibody and then were quantified using Fiji (https://imagej.net/).

375 The RLUs were then normalized against the amounts of pseudovirus-packaged spikes.

376 All of the measurements were carried out in quadruplicates.

377 For pseudovirus entry inhibition, mouse sera were serially diluted in DMEM

378 media and then mixed with SARS-CoV-2 pseudoviruses. Subsequently the mixtures were

379 added to HEK293T cells expressing human ACE2 for the pseudovirus entry assay. The

380 fitted curves and the $50 \%$ neutralizing antibody titers $\left(\mathrm{NT}_{50}\right)$ were calculated using the

381 Graphpad Prism program. All the measurements were carried out in triplicates.

382 Western blot

383 Pseudoviruses were mixed with SDS loading buffer and then were incubated at

$38495^{\circ} \mathrm{C}$ for $10 \mathrm{~min}$. Samples were run in a $10 \%$ SDS Tris-Glycine Gel and transferred to a 
385 PVDF membrane. An anti-c9 or anti-His ${ }_{6}$ monoclonal primary antibody (1:1000 dilution,

386 Santa Cruz Biotech) and a horseradish peroxidase-conjugated mouse secondary antibody

387 (1:10,000 dilution, Jackson Laboratory) were used for Western blotting. A LAS-4000

388 imager was used to develop images.

389 Protein pull-down assay

$390 \quad$ Protein pull-down assay was performed using a Dynabeads immunoprecipitation

391 kit (Invitrogen) as previously described (41). Briefly, $10 \mu \mathrm{L}$ of Dynabeads, either for

392 His $_{6}$-tagged proteins or for Fc-tagged proteins, were washed with PBS buffer and then

393 were incubated with either $8 \mu \mathrm{g}$ ACE2-His (human ACE2 with a C-terminal His ${ }_{6}$ tag) or

$39410 \mu \mathrm{g}$ ACE2-Fc (human ACE2 with a C-terminal Fc tag), respectively. Subsequently,

395 ACE2-bound beads were washed with PBS buffer plus 0.05\% Tween-20 (PBST) and

396 then were aliquoted into different tubes for later use. To prepare cell-associated

397 coronavirus spike, HEK293T cells were transfected with pcDNA3.1(+) plasmid encoding

398 coronavirus spike (containing a C-terminal c9 tag). $48 \mathrm{~h}$ after transfection, the spike-

399 expressing cells were lysed in immunoprecipitation assay buffer using a sonicator and

400 then centrifuged. The supernatants containing solubilized coronavirus spike (or purified

401 recombinant coronavirus RBDs for RBD pull-down assay) were incubated with the

402 ACE2-bound beads (spike or RBD was in excess of ACE2). Then beads were washed

403 with PBST buffer, and the bound proteins were eluted using elution buffer. The samples

404 were then subjected to Western blot analysis and detected using an anti-C9 tag antibody

405 or anti-His 6 tag antibody.

406 Cryo-electron microscopy (cryo-EM) 
For sample preparation, aliquots of recombinant SARS-CoV-2 spike ectodomain

$408(3 \mu \mathrm{l} ; 0.35 \mathrm{mg} / \mathrm{ml}$; in buffer containing $10 \mathrm{mM}$ Tris $\mathrm{pH} 7.4$ and $100 \mathrm{mM} \mathrm{NaCl})$ were

409 applied to glow-discharged CF-2/1-4C C-flat grids (Protochips). The grids were then

410 plunge-frozen in liquid ethane using a Vitrobot system (FEI Company).

411 For data collection, images were recorded using a Gatan K2 Summit direct

412 electron detector in super resolution mode, attached to a FEI Titan-Krios TEM. The

413 automated software SerialEM was used to collect movies at 22,500x magnification and at

414 a defocus range between 0.6 and $2.6 \mu \mathrm{m} .1847$ movies were collected for FnM-point

415 spike ectodomain, 4784 movies were collected for FnM-deletion spike ectodomain and

4164563 movies were collected for K417V/FnM-deletion spike ectodomain. Each movie had

417 an exposure of $7.822 \mathrm{e}-/ \AA 2 / \mathrm{s}$ fractionated in 40 frames of 8 second exposure. Data

418 collection statistics are summarized in Table S2.

419 For data processing, whole frames in each movie were corrected for motion and

420 dose compensation using MotionCor2 (43). The final images were bin-averaged to reach

421 a pixel size of $1.04 \AA$. The parameters of the microscope contrast transfer function were

422 estimated for each micrograph using GCTF (44). Particles were automatically picked and

423 extracted using Gautomatch (http://www.mrc-lmb.cam.ac.uk/kzhang/Gautomatch/) and

424 RELION (45) with a box size of 300 pixels. For FnM-deletion spike ectodomain, 728,804

425 particles were initially extracted and subjected to 2D alignment and clustering using

426 RELION. The best classes were then selected for an additional 2D alignment. $~ 5,000$ best

427 particles were selected for creating the initial 3D model using RELION. 107,268 particles

428 selected from 2D alignment were then subjected to 3D classification. The best class with

42965,302 particles was subjected to $3 \mathrm{D}$ refinement to generate the final density map with 
430 C3 symmetry. For FnM-point spike ectodomain, 583,127 particles were initially

431 extracted and subjected to 2D alignment and clustering using RELION. The best classes

432 were then selected for an additional 2D alignment. 5000 best particles were selected for

433 creating the initial 3D model using RELION. 52,134 particles selected from 2D

434 alignment were then subjected to 3D classification for open and closed conformations.

435 The best open-conformation class with 21,894 particles and the best closed-conformation

436 class with 23,849 particles were subjected to 3D refinement to generate the final density

437 maps with C1 symmetry and C3 symmetry, respectively. For K417V/FnM-deletion spike

438 ectodomain, 1,267,763 particles were initially extracted and subjected to 2D alignment

439 and clustering using RELION. The best classes were then selected for an additional 2D

440 alignment. 124,721 best particles were selected for creating the initial 3D model using

441 RELION. 26,126 particles selected from 2D alignment were then subjected to 3D

442 classification for open and closed conformations. The best open-conformation class with

443101,413 particles and the best closed-conformation class with 9,502 particles were

444 subjected to 3D refinement to generate the final density maps with C1 symmetry and C3

445 symmetry, respectively. The final density maps were sharpened with modulation transfer

446 function of $\mathrm{K} 2$ operated at $300 \mathrm{keV}$ using RELION. Reported resolutions were based on

447 the gold standard Fourier shell correlation $(\mathrm{FSC})=0.143$ criterion. Fourier shell

448 correction curves were corrected for the effects of soft masking by high-resolution noise

449 substitution (46).

$450 \quad$ Model building and refinement

451 The initial model of the SARS-CoV-2 spike ectodomain was obtained by fitting

452 the cryo-EM structure of a previously determined SARS-CoV-2 FnM-point spike 
453 ectodomain (PDB ID: 6VXX) into our cryo-EM density maps using UCSF Chimera and

454 Coot $(47,48)$. Manual model rebuilding was performed using Coot based on the well-

455 defined continuous density of the main chain. Side chain assignments were guided

456 through the density of bulky amino acid residues. The structural model of SARS-CoV-2

457 spike ectodomain was refined using Phenix (49) with geometry restrains and three-fold

458 noncrystallographic symmetry constraints. Refinement and model rebuilding were carried

459 out iteratively until no further improvements were achieved in geometry parameters and

460 model-map correlation coefficient. The quality of the final model was analyzed using

461 MolProbity (50). The validation statistics of the structural models are summarized in

462 Table S2.

463 Calculation of interface area

464 The buried surface areas between NTD and RBD and between RBD and RBD in

465 the trimeric spike ectomains were calculated using the PISA server at the European

466 Bioinformatics Institute (http://www.ebi.ac.uk/pdbe/prot int/pistart.html) (51). For each

467 trimeric spike ectodomain, a PDB file containing the coordinates from the pair of the

468 corresponding domains was submitted to the PISA server, and the buried surface area for

469 each pair was individually calculated.

$470 \quad$ Calculation of angle between domains

471 The rotation angle between the S1 domains in SARS-CoV-2 spike structures was

472 calculated using the angle_between_domains script in the Psico program

473 (https://pymolwiki.org/index.php/Psico).

474 Mouse immunization 
Male and female BALB/c mice were intramuscularly (I.M.) immunized with each

476 recombinant SARS-CoV-2 spike ectodomain (10 $\mu \mathrm{g} /$ mouse; 4 mice/group), or PBS

477 buffer, in the presence of two adjuvants: aluminum hydroxide (Alum, $500 \mu \mathrm{g} / \mathrm{mouse}$;

478 InvivoGen) and monophosphoryl lipid A (MPL, $10 \mu \mathrm{g} / \mathrm{mouse}$; InvivoGen). The mice

479 were boosted once via I.M. with the same immunogen at 4 weeks. Mouse sera were

480 collected 10 days after the $2^{\text {nd }}$ immunization and detected for antibody responses against

481 the RBD and neutralizing antibodies against SARS-CoV-2 pseudovirus entry.

482 ELISA

483 ELISA was carried out to detect the serum IgG antibodies targeting the RBD.

484 Briefly, ELISA plates were coated with recombinant SARS-CoV-2 RBD (containing a C-

485 terminal His tag) $(1 \mu \mathrm{g} / \mathrm{ml})$ at $4^{\circ} \mathrm{C}$ overnight, and blocked with $2 \%$ fat-free milk at $37^{\circ} \mathrm{C}$

486 for $2 \mathrm{~h}$. After three washes with wash buffer (PBS $+0.1 \%$ Tween-20), the ELISA plates

487 were incubated with each individual mouse serum at serial dilutions. After incubation at

$48837^{\circ} \mathrm{C}$ for $1 \mathrm{~h}$, the ELISA plates were washed, followed by addition of a horseradish

489 peroxidase-conjugated mouse secondary antibody (1:5,000) (Thermo Fisher Scientific).

490 After another incubation at $37^{\circ} \mathrm{C}$ for $1 \mathrm{~h}$, ELISA substrate (Sigma-Aldrich) was added.

491 The ELISA reaction was stopped using $1 \mathrm{~N} \mathrm{H}_{2} \mathrm{SO}_{4}$, and the ELISA signal was read at the

$492450 \mathrm{~nm}$ wavelength using an ELISA plate reader (Tecan).

493 Ethics statement

$494 \quad$ Mouse work was performed in strict accordance with the guidance and

495 recommendations in the Guide for the Care and Use of Laboratory Animals (National

496 Research Council Institute for Laboratory Animal Research). Experiments were 
497 conducted under animal use protocols approved by the Institutional Animal Care and Use

498 Committees at the New York Blood Center.

499

500

501 


\section{References}

503 1. Q. Li et al., Early Transmission Dynamics in Wuhan, China, of Novel

$504 \quad$ Coronavirus-Infected Pneumonia. N Engl J Med, (2020).

505 2. C. Huang et al., Clinical features of patients infected with 2019 novel coronavirus

506 in Wuhan, China. Lancet, (2020).

507 3. N. Lee et al., A major outbreak of severe acute respiratory syndrome in Hong

508 Kong. New England Journal of Medicine 348, 1986-1994 (2003).

509 4. J. S. M. Peiris et al., Coronavirus as a possible cause of severe acute respiratory

$510 \quad$ syndrome. Lancet 361, 1319-1325 (2003).

511 5. R. A. M. Fouchier et al., A previously undescribed coronavirus associated with 512 respiratory disease in humans. Proceedings of the National Academy of Sciences $513 \quad$ of the United States of America 101, 6212-6216 (2004).

514 6. L. van der Hoek et al., Identification of a new human coronavirus. Nature $515 \quad$ Medicine 10, 368-373 (2004).

516 7. F. Wu et al., Neutralizing antibody responses to SARS-CoV-2 in a COVID-19

517 recovered patient cohort and their implications. medRxiv, $518 \quad 2020.2003 .2030 .20047365(2020)$.

519 8. F. Zhou et al., Clinical course and risk factors for mortality of adult inpatients

9. R. Woelfel et al., Clinical presentation and virological assessment of hospitalized cases of coronavirus disease 2019 in a travel-associated transmission cluster. medRxiv, 2020.2003.2005.20030502 (2020).

10. M. Gao et al., A study on infectivity of asymptomatic SARS-CoV-2 carriers. Respiratory medicine 169, 106026 (2020).

11. A. Kronbichler et al., Asymptomatic patients as a source of COVID-19 infections: A systematic review and meta-analysis. International journal of infectious diseases : IJID : official publication of the International Society for Infectious Diseases 98, 180-186 (2020).

534 13. F. Li, Receptor recognition and cross-species infections of SARS coronavirus. 535 Antiviral Res 100, 246-254 (2013). 
536 14. J. Huynh et al., Evidence supporting a zoonotic origin of human coronavirus 537 strain NL63. J Virol 86, 12816-12825 (2012).

538 15. X. Y. Ge et al., Isolation and characterization of a bat SARS-like coronavirus that uses the ACE2 receptor. Nature 503, 535-538 (2013).

540 16. Y. Guan et al., Isolation and characterization of viruses related to the SARS coronavirus from animals in Southern China. Science 302, 276-278 (2003).

542 17. P. Zhou et al., A pneumonia outbreak associated with a new coronavirus of 543 probable bat origin. Nature, (2020).

544 18. F. Li, Structure, Function, and Evolution of Coronavirus Spike Proteins. Annual 545 review of virology 3, 237-261 (2016).

546 19. L. Y. Du et al., The spike protein of SARS-CoV - a target for vaccine and 547 therapeutic development. Nature Reviews Microbiology 7, 226-236 (2009).

548 20. F. Li, Receptor recognition mechanisms of coronaviruses: a decade of structural 549

21. S. Belouzard, J. K. Millet, B. N. Licitra, G. R. Whittaker, Mechanisms of coronavirus cell entry mediated by the viral spike protein. Viruses 4, 1011-1033 (2012).

22. T. Heald-Sargent, T. Gallagher, Ready, set, fuse! The coronavirus spike protein and acquisition of fusion competence. Viruses 4, 557-580 (2012).

555 23. W. H. Li et al., Angiotensin-converting enzyme 2 is a functional receptor for the 556

26. J. Shang et al., Structural basis of receptor recognition by SARS-CoV-2. Nature

565 27. J. Shang et al., Cell entry mechanisms of SARS-CoV-2. Proc Natl Acad Sci U S A $566 \quad \mathbf{1 1 7}, 11727-11734(2020)$.

567 28. A. C. Walls et al., Structure, Function, and Antigenicity of the SARS-CoV-2 $568 \quad$ Spike Glycoprotein. Cell, (2020). 
29. Z. Ke et al., Structures and distributions of SARS-CoV-2 spike proteins on intact virions. Nature, (2020).

571

572

573

574

575

576

577

578

579

580

581

582

583

584

585

586

587

588

589

590

591

592

593

594

595

596

597

598

599

600

601

602

30. A. C. Walls et al., Glycan shield and epitope masking of a coronavirus spike protein observed by cryo-electron microscopy. Nat Struct Mol Biol 23, 899-905 (2016).

31. A. G. Wrobel et al., SARS-CoV-2 and bat RaTG13 spike glycoprotein structures inform on virus evolution and furin-cleavage effects. Nat Struct Mol Biol 27, 763767 (2020).

32. M. Gui et al., Cryo-electron microscopy structures of the SARS-CoV spike glycoprotein reveal a prerequisite conformational state for receptor binding. Cell Res 27, 119-129 (2017).

33. J. Shang et al., Structure of mouse coronavirus spike protein complexed with receptor reveals mechanism for viral entry. PLoS Pathog 16, e1008392 (2020).

34. X. Xiong et al., A thermostable, closed SARS-CoV-2 spike protein trimer. Nat Struct Mol Biol 27, 934-941 (2020).

35. Y. Cai et al., Distinct conformational states of SARS-CoV-2 spike protein. Science 369, 1586-1592 (2020).

36. J. K. Millet, G. R. Whittaker, Host cell proteases: Critical determinants of coronavirus tropism and pathogenesis. Virus Res 202, 120-134 (2015).

37. L. Yurkovetskiy et al., Structural and Functional Analysis of the D614G SARSCoV-2 Spike Protein Variant. Cell 183, 739-751.e738 (2020).

38. S. Ozono et al., SARS-CoV-2 D614G spike mutation increases entry efficiency with enhanced ACE2-binding affinity. Nature communications 12, 848 (2021).

39. Y. J. Hou et al., SARS-CoV-2 D614G variant exhibits efficient replication ex vivo and transmission in vivo. Science 370, 1464-1468 (2020).

40. K. Wu et al., A Virus-Binding Hot Spot on Human Angiotensin-Converting Enzyme 2 Is Critical for Binding of Two Different Coronaviruses. Journal of Virology 85, 5331-5337 (2011).

41. Y. Wan et al., Molecular Mechanism for Antibody-Dependent Enhancement of Coronavirus Entry. Journal of Virology 94, e02015-02019 (2020).

42. G. Peng et al., Structural and Molecular Evidence Suggesting Coronavirus-driven Evolution of Mouse Receptor. J Biol Chem 292, 2174-2181 (2017).

43. X. Li et al., Electron counting and beam-induced motion correction enable nearatomic-resolution single-particle cryo-EM. Nature methods 10, 584-590 (2013). 
603 44. K. Zhang, Getf: Real-time CTF determination and correction. Journal of 604 structural biology 193, 1-12 (2016).

605 45. S. H. Scheres, RELION: implementation of a Bayesian approach to cryo-EM 606 structure determination. Journal of structural biology 180, 519-530 (2012).

607 46. S. Chen et al., High-resolution noise substitution to measure overfitting and 608 validate resolution in 3D structure determination by single particle electron $609 \quad$ cryomicroscopy. Ultramicroscopy 135, 24-35 (2013).

610 47. T. D. Goddard, C. C. Huang, T. E. Ferrin, Visualizing density maps with UCSF

48. P. Emsley, K. Cowtan, Coot: model-building tools for molecular graphics. Acta

614 49. P. D. Adams et al., PHENIX: a comprehensive Python-based system for macromolecular structure solution. Acta Crystallographica Section D-Biological Crystallography 66, 213-221 (2010).

617 50. V. B. Chen et al., MolProbity: all-atom structure validation for macromolecular

51. E. Krissinel, K. Henrick, Inference of macromolecular assemblies from crystalline state. J Mol Biol 372, 774-797 (2007).

622 


\section{Figure legends:}

626 Figure 1: Molecular switch for SARS-CoV-2 spike to close down. (A) One-

627 dimensional schematic representation of SARS-CoV-2 spike. NTD: N-terminal domain.

628 RBD: receptor-binding domain. SD1: subdomain 1. SD2: subdomain 2. TM:

629 transmembrane anchor. IC: intracellular tail. Furin cleavage site is indicated by arrow.

630 (B) Three-dimensional schematic representation of SARS-CoV-2 spike in the pre-fusion

631 structure. The double curve arrow indicates a mixture of open and closed spikes. Double

632 dotted lines represent viral envelope. Arrows indicate location of furin motif (FnM). Also

633 listed is the comparison of the sequences in the furin motif region among SARS-CoV-2,

634 RaTG13-CoV and SARS-CoV-1 spikes. SARS-CoV-2 (FnM-point) spike contains point

635 mutations in FnM. SARS-CoV-2 (FnM-deletion) spike contains FnM deletion as in

636 SARS-CoV-1. SARS-CoV-2 (FnM-deletion-2) spike contains FnM deletion as in

637 RaTG13-CoV. (C) Protein pull-down assay using recombinant human ACE2 as the bait 638 and cell-associated SARS-CoV-2 spike molecules (wild type and mutants) as the targets.

639 Protein levels were detected using Western blot. Top, cell-expressed SARS-CoV-2 spike.

640 Middle, pull-down results using His 6 -tagged ACE2. Bottom, pull-down results using Fc-

641 tagged ACE2. The expression of SARS-CoV-2 spike (which contained a C-terminal c9

642 tag) was detected using an anti-c9 antibody. Mock, no spike. WT, wild type. (D) SARS-

643 CoV-2 pseudovirus entry into human ACE2-expressing cells. Top, pseudovirus entry

644 efficiency normalized against the expression of the spike (see bottom). Entry efficiency

645 of wild type pseudoviruses was taken as 100\%. Bottom, SARS-CoV-2 spike (which

646 contained a C-terminal c9 tag) packaged in pseudoviruses. Its expression was detected by

647 Western blot using an anti-c9 antibody. Individual data points are shown as dots. A 
648 comparison (two-tailed Student's t-test) was performed on data between different groups

$649(\mathrm{n}=8) . * * * \mathrm{P}<0.001$. All experiments were repeated independently three times with

650 similar results.

651 Figure 1 - Source Data 1: gels/blots

652

653 Figure 2: Cryo-EM analyses of the conformations of recombinant SARS-CoV-2

654 spike ectodomain mutants. These spike mutants contain furin motif deletion (A), furin

655 motif point mutations (B), or both K17V mutation and furin motif deletion (C),

656 respectively. Details of these mutations were explained in Fig. 1B. Their EM density

657 maps, corresponding resolution, and distribution of the particles in open and closed

658 conformations are shown. Atomic models were built for the closed spikes containing

659 furin motif deletion and furin motif point mutations, respectively. Three-dimensional

660 schematic representations are shown for the other spike particles.

661

662 Figure 3: Molecular switch for RaTG13-CoV spike to open up. (A) Three-

663 dimensional schematic representation of RaTG13-CoV spike in the pre-fusion structure

664 with closed RBDs. RaTG13-CoV (FnM-insert) spike contains inserted FnM as in SARS-

665 CoV-2. RaTG13-CoV (GSGS-insert) spike contains an inserted GSGS sequence in the

666 same location as FnM. (B) Protein pull-down assay using recombinant human ACE2 as

667 the bait and cell-associated RaTG13-CoV spike molecules as the targets. Top, cell-

668 expressed RaTG13-CoV spike. Middle, pull-down results using His6-tagged ACE2.

669 Bottom, pull-down results using Fc-tagged ACE2. (C) RaTG13-CoV pseudovirus entry

670 into human ACE2-expressing cells. Top, pseudovirus entry efficiency normalized against 
671 the expression level of the spike (see bottom). Bottom, RaTG13-CoV spike packaged in

672 pseudoviruses. Data are mean + S.E.M. A comparison (two-tailed Student's t-test) was

673 performed on data between different groups $(\mathrm{n}=4) . * * * \mathrm{P}<0.001$. All experiments were

674 repeated independently three times with similar results.

675 Figure 3 - Source Data 1: gels/blots

676

677 Figure 4: Molecular switch for SARS-CoV-2 spike to open up. (A) Identification of a

678 critical residue Lys417 in SARS-CoV-2 spike that stabilizes the RBD in the closed

679 conformation. The corresponding residue is a valine in SARS-CoV-1. The structure of

680 the closed SARS-CoV-2 spike (PDB 6VXX) is presented from a side view to show three

681 packed RBDs. Each monomeric subunit of the spike trimer is colored differently. (B)

682 Protein pull-down assay using recombinant human ACE2 as the bait and cell-associated

683 SARS-CoV-2 spike molecules as the targets. Top, cell-expressed SARS-CoV-2 spike.

684 Middle, pull-down results using His 6 -tagged ACE2. Bottom, pull-down results using Fc-

685 tagged ACE2. (C) SARS-CoV-2 pseudovirus entry into human ACE2-expressing cells.

686 Top, pseudovirus entry efficiency normalized against the expression level of the spike

687 (see bottom). Bottom, SARS-CoV-2 spike in packaged pseudoviruses. Data are mean +

688 S.E.M. A comparison (two-tailed Student's t-test) was performed on data between

689 different groups $(\mathrm{n}=8) . * * * \mathrm{P}<0.001$. All experiments were repeated independently three

690 times with similar results.

691 Figure 4 - Source Data 1: gels/blots

692 
693 Figure 5: Immune evasion of closed SARS-CoV-2 spike. Mice were immunized with

694 one of the mutant SARS-CoV-2 spikes (4 mice in each group). Subsequently the mouse

695 sera were assayed for titers of RBD-targeting antibodies and neutralizing antibodies.

696 Buffer PBS was used as a negative control in mouse immunization. (A) ELISA for

697 detecting the titers of RBD-targeting IgG antibodies. SARS-CoV-2 RBD (containing a C-

698 terminal $\mathrm{His}_{6}$ tag) was coated on ELISA plates, and serially diluted sera from each

699 immunized mouse were added for detection of RBD/IgG binding. The titers were

700 expressed as the endpoint dilutions that remain positively detectable. A titer was

701 determined for sera from each immunized mouse. Data are mean + S.E.M. A comparison

702 (two-tailed Student's t-test) was performed on sera between the FnM-deletion group and

703 one of the other experimental mouse groups $(\mathrm{n}=4) . * * \mathrm{P}<0.01 . * \mathrm{P}<0.05$. (B)

704 Pseudovirus entry inhibition assay for detecting the titers of neutralizing antibodies.

705 SARS-CoV-2 pseudoviruses were used to enter human ACE2-expressing cells in the

706 presence of serially diluted sera from each group of immunized mice (sera from mice

707 within each immunization group were pooled together for this assay). $\mathrm{NT}_{50}$ of sera was

708 determined as the dilution factor that led to $50 \%$ inhibition of pseudovirus entry. High

$709 \mathrm{NT}_{50}$ suggests high titers of neutralizing antibodies in the sera. Data are mean + S.E.M.

710 A comparison (two-tailed Student's t-test) was performed on sera at individual dilution

711 point between the FnM-deletion group and one of the other experimental mouse groups

$712(\mathrm{n}=3) . * * * \mathrm{P}<0.001 . * * \mathrm{P}<0.01 . * \mathrm{P}<0.05$. All experiments were repeated independently

713 three times with similar results.

714

715 


\section{Supplementary figure legends}

717 Figure S1: Cryo-EM classifications of SARS-CoV-2 spike particles. (A)

718 Representative 2D class averages in different orientations. (B) Summary of 3D

719 classifications of SARS-CoV-2 spike particles.

720

721 Figure S2: Cryo-EM data for two mutant SARS-CoV-2 spikes whose atomic models

722 were built. (A) Gold-standard Fourier shell correlation (FSC) curves. The resolutions

723 were set at $3.8 \AA$ for FnM-deletion spike and $4.37 \AA$ for FnM-point spike. The 0.143

724 cutoff value is indicated by horizontal dotted line. (B) Partial cryo-EM density maps with

725 fitted atomic models for FnM-deletion spike (model in blue) and for FnM-point spike

726 (model in red).

727

728 Figure S3: S1 packing in SARS-CoV-2 spike. (A) Structure of trimeric S1 in FnM-

729 deletion spike. Three subunits are colored differently. Noted in parentheses are the

730 monomeric subunits where each structural element is located. (B) Comparison of S1

731 packing in two cryo-EM structures determined in the current study (FnM-deletion spike

732 in the closed conformation and FnM-point spike in the closed conformation) and another

733 cryo-EM structure determined in a previous study (FnM-point spike in the closed

734 conformation; PDB: 6VXX).

735

736 Figure S4: Role of furin motif loop in S1 packing (A) Comparison of chain traces of

737 monomeric S1 in FnM-deletion spike (colored in blue) and that in FnM-point spike

738 (colored in red). (B) Comparison of chain traces of monomeric S1 in FnM-deletion spike 
739 (colored in red) and that in mouse hepatitis coronavirus (MHV) spike (colored in red;

740 PDB: 6VSJ).

741 
A Furin motif deletion

\section{$3.8 \AA$}

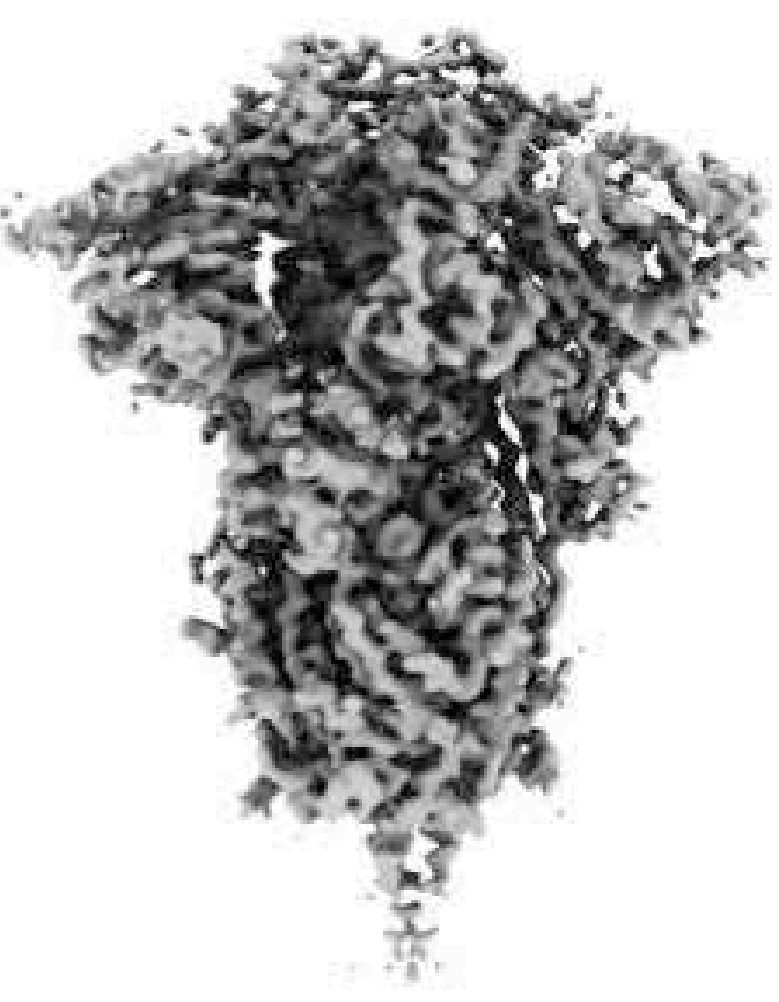

Closed (100\%)

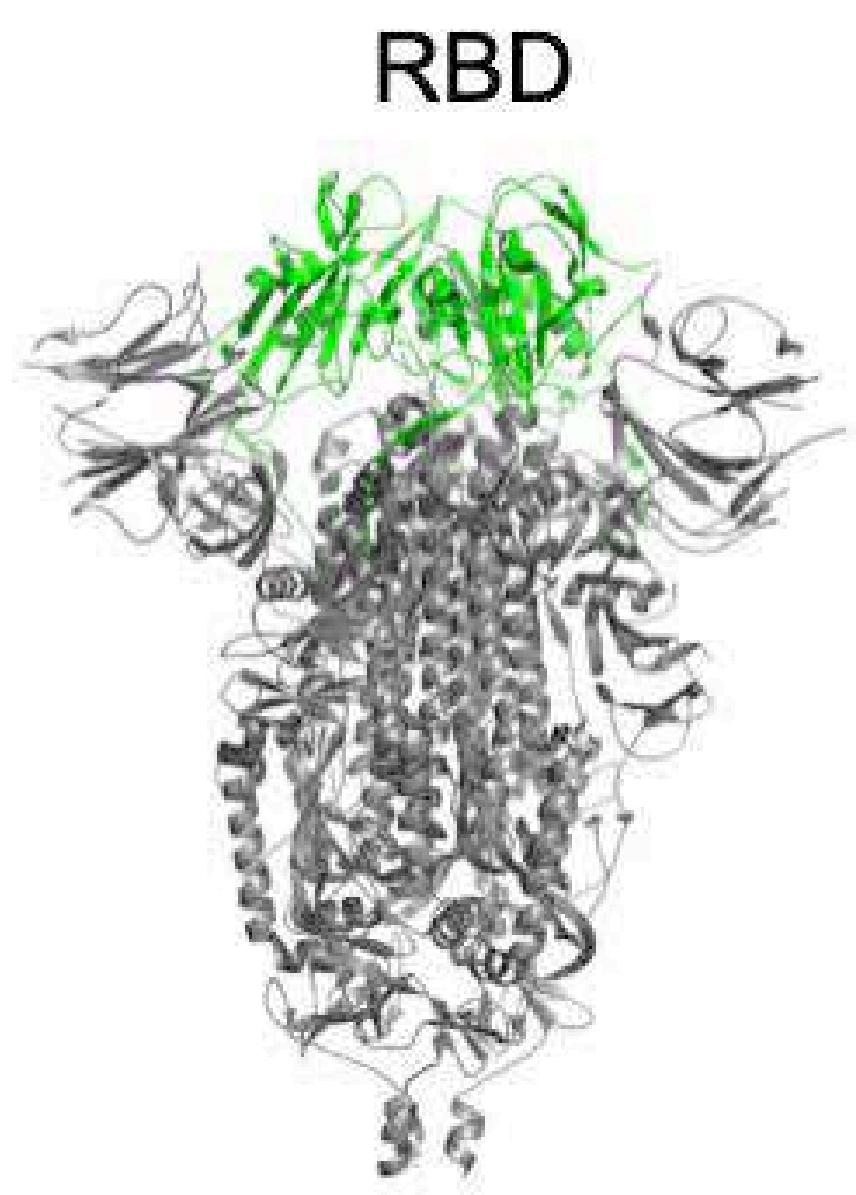

B

Furin motif point mutations

\section{C}

RBD mutation (K417V)

+ Furin motif deletion

$4.4 \AA$

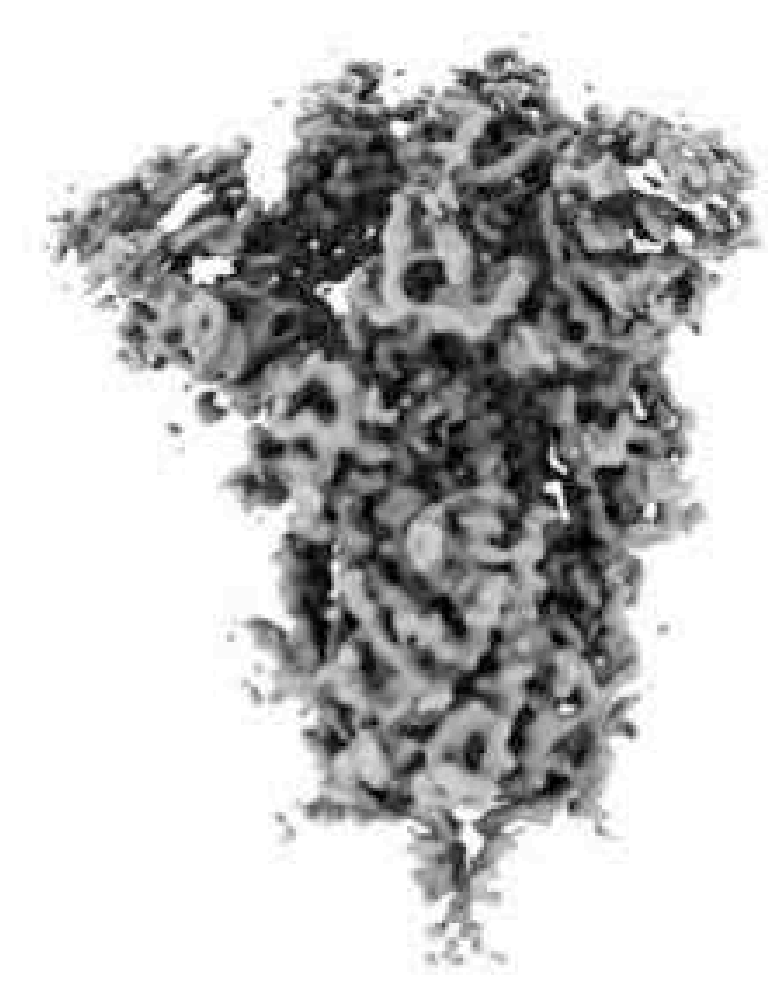

Closed (52\%)

RBD

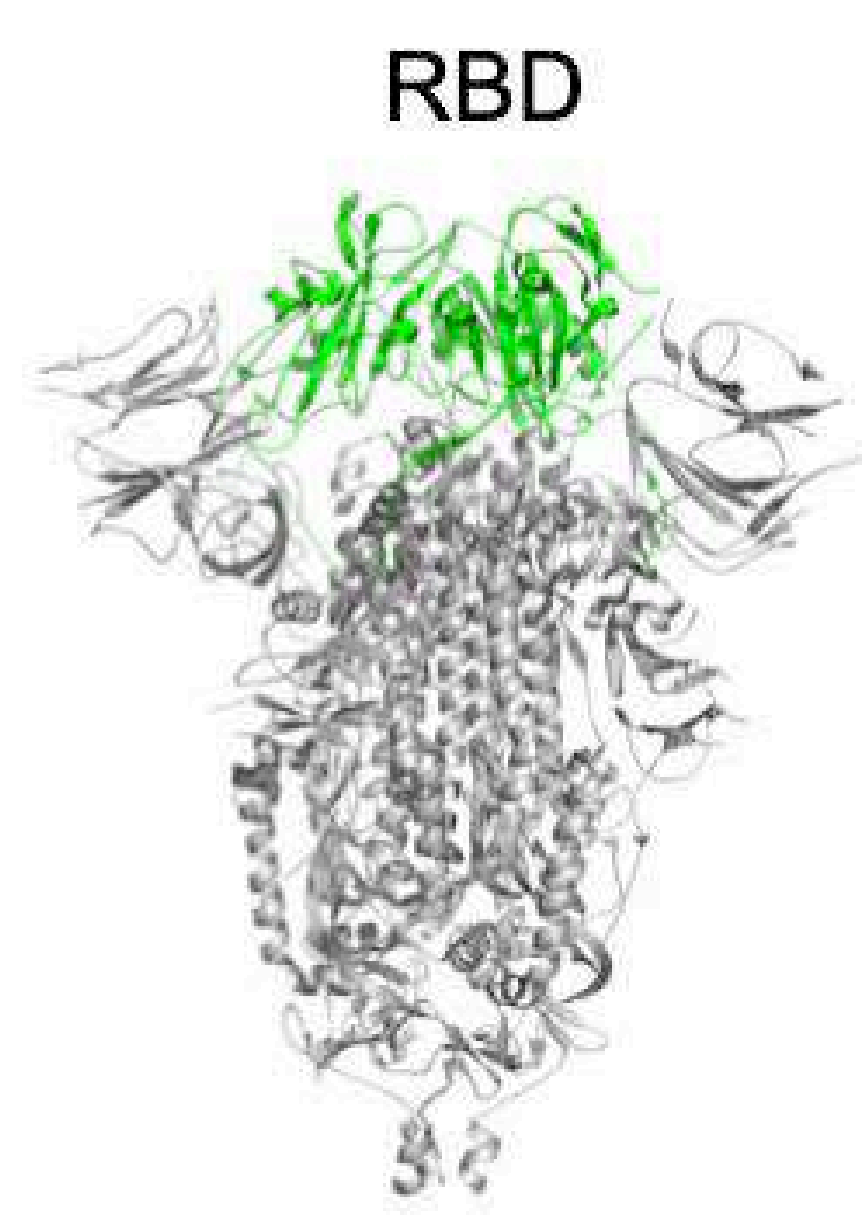

$5.3 \AA$

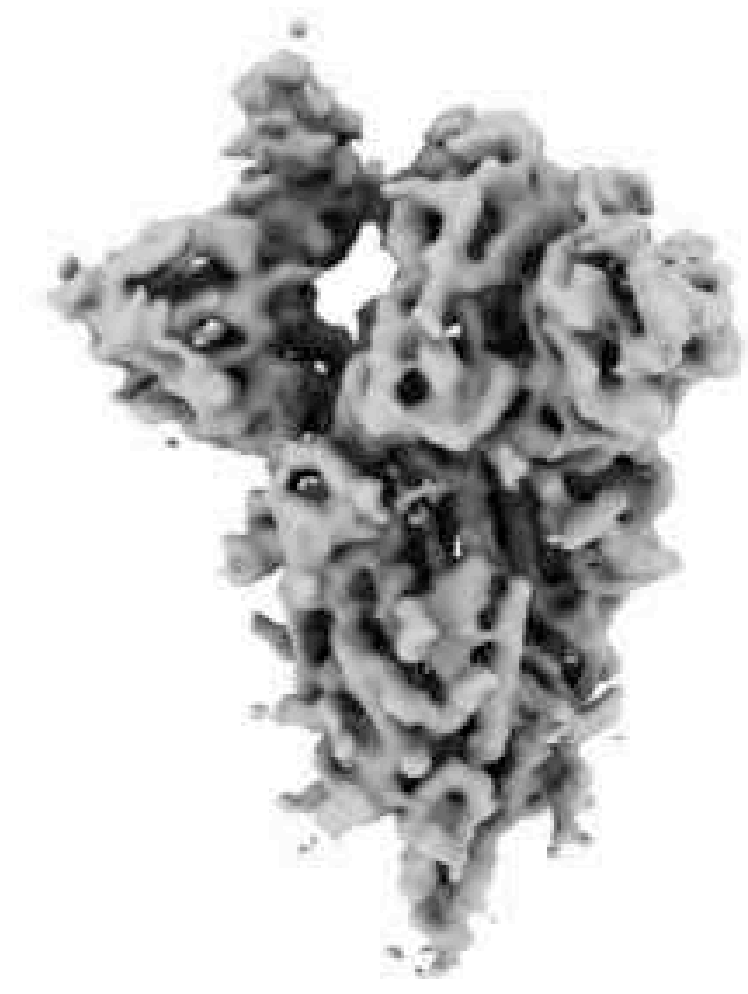

Open (48\%)

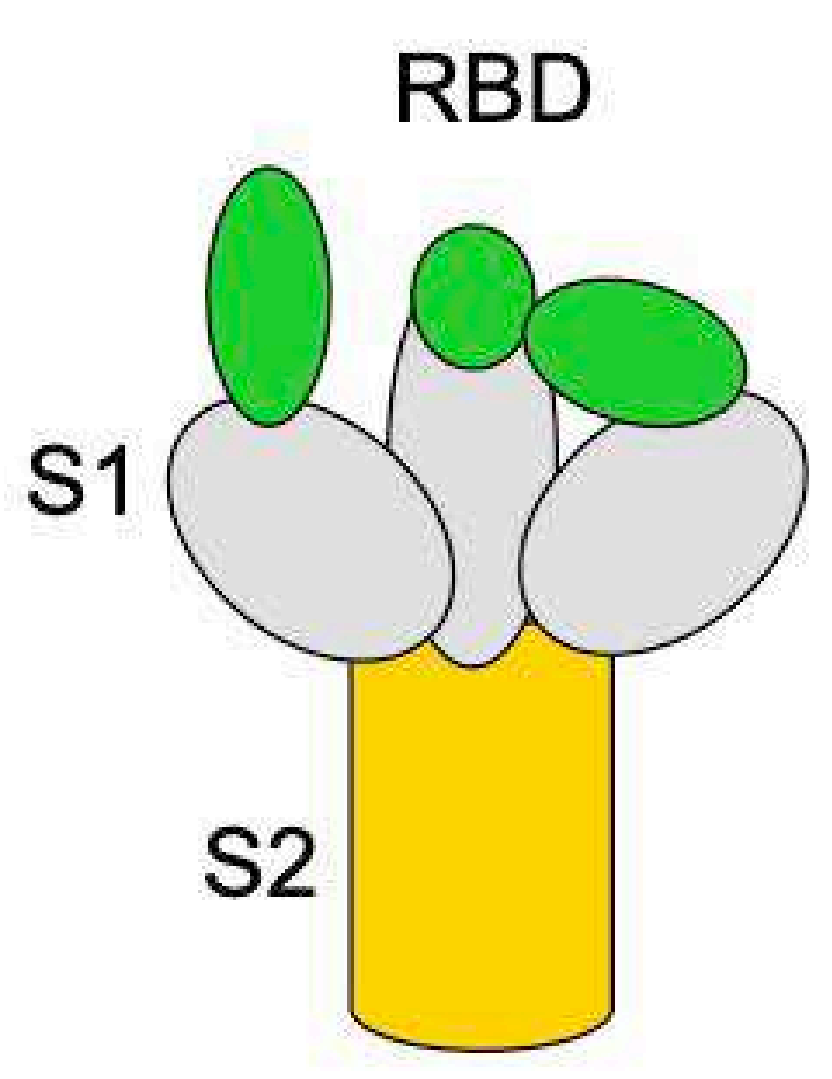

Figure 2

$4.6 \AA$ $4.6 \AA$

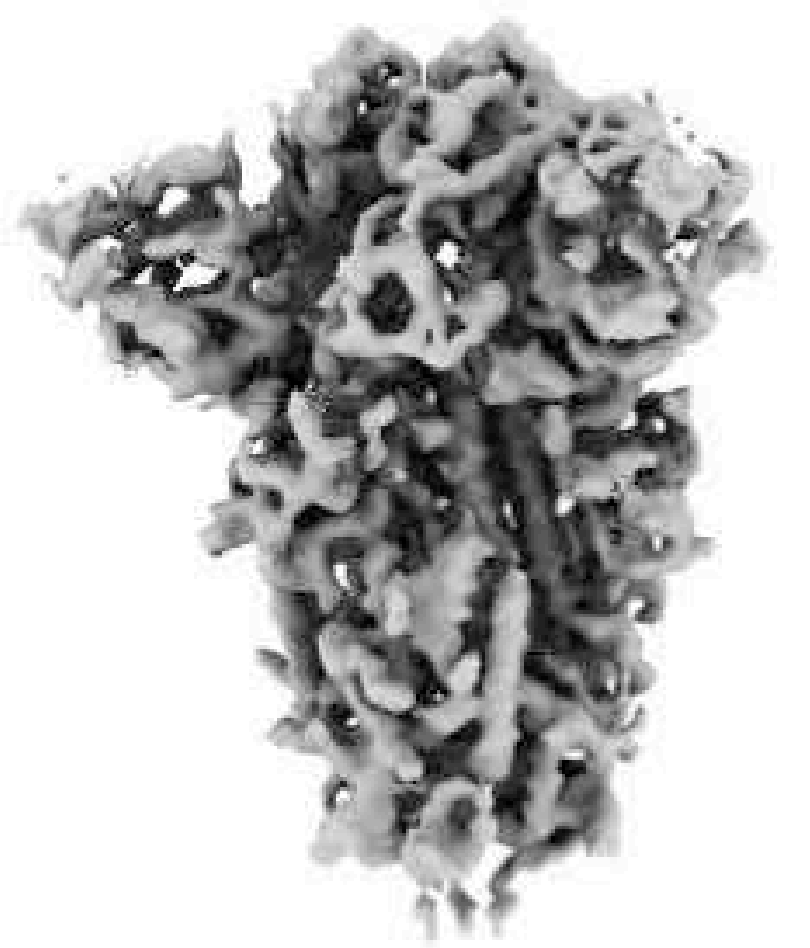

Closed (9\%)
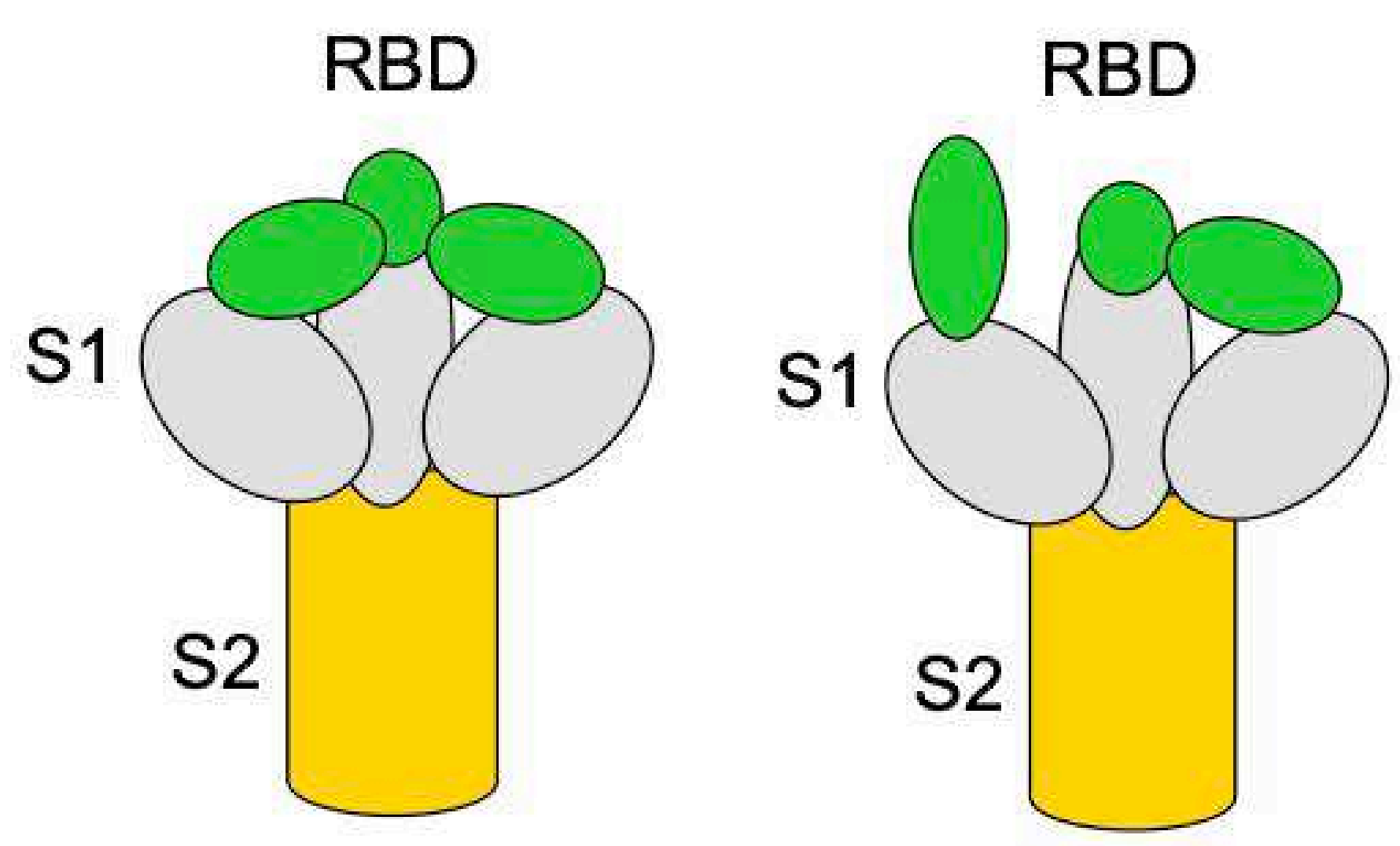

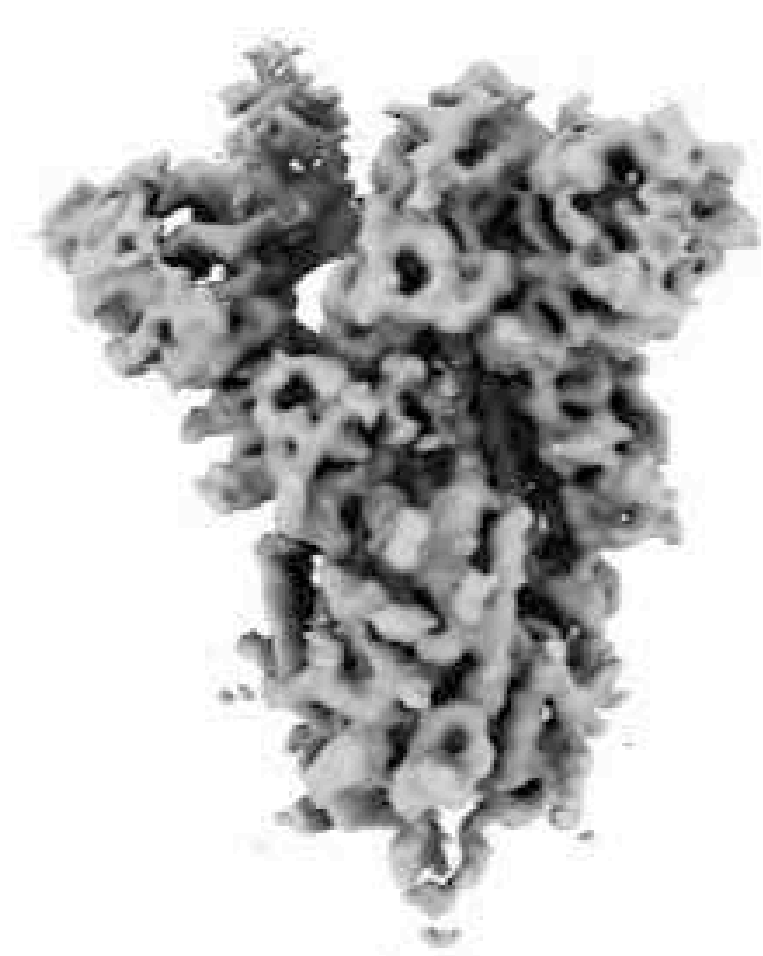

Open (91\%) 
A

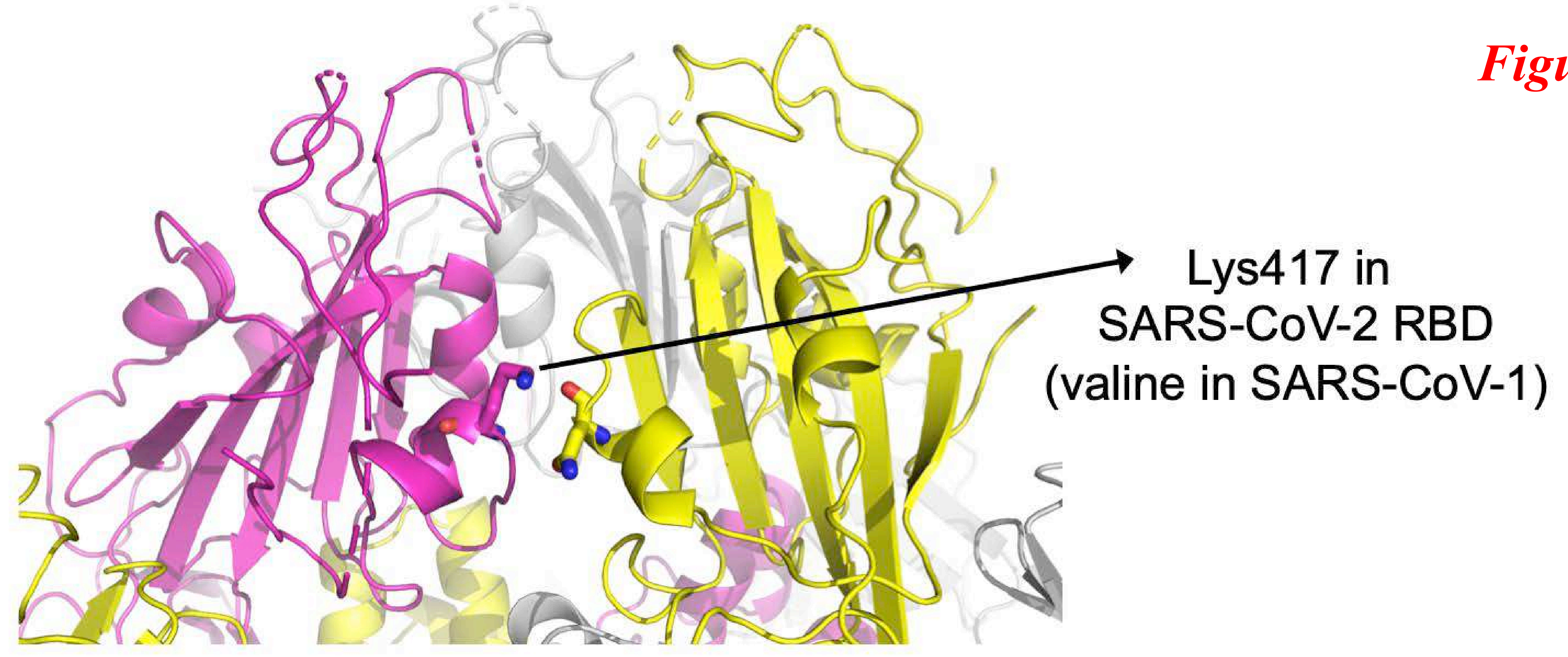

B

Pull down of cell-surface SARS-CoV-2 spike
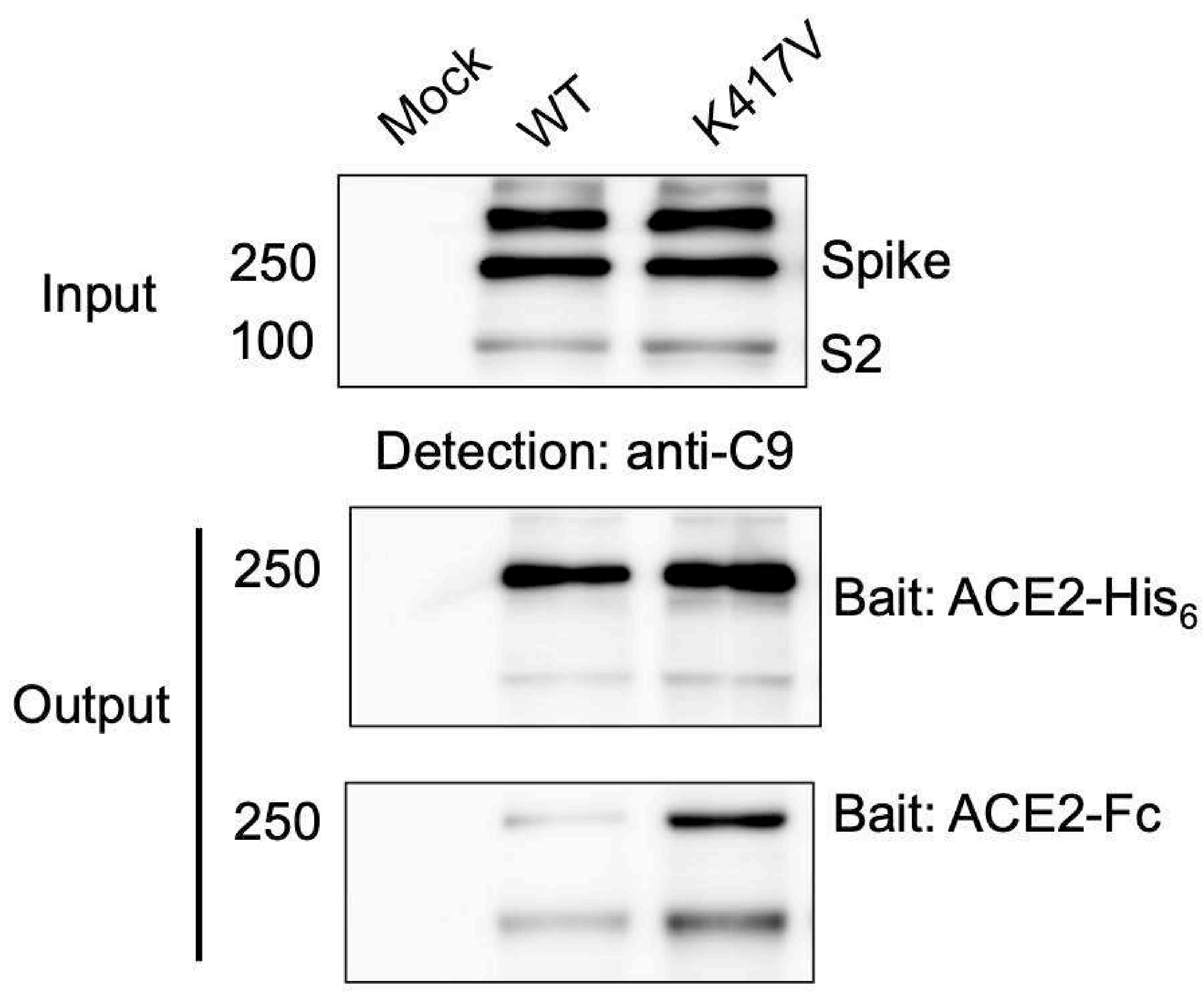

C SARS-CoV-2 pseudovirus

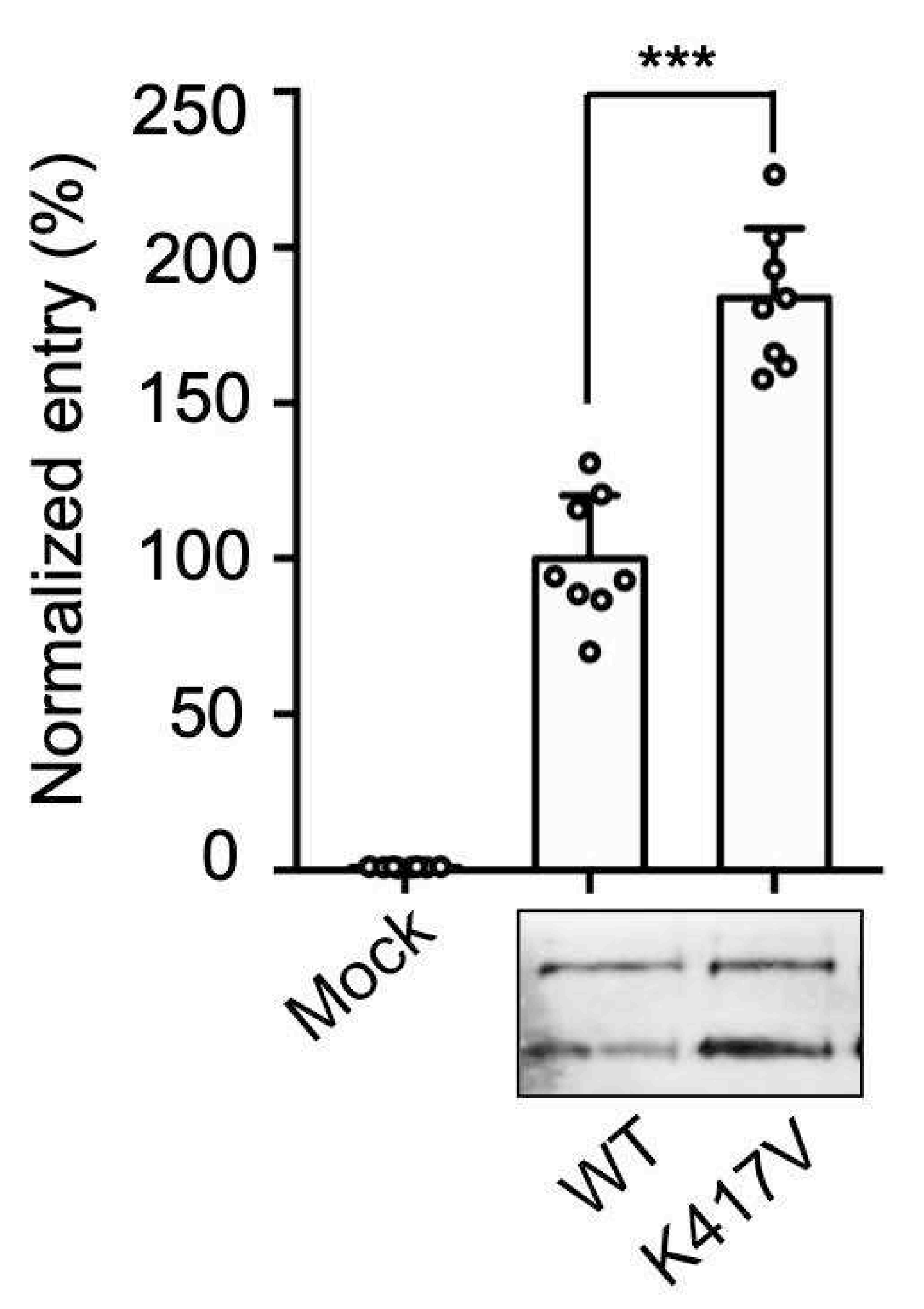



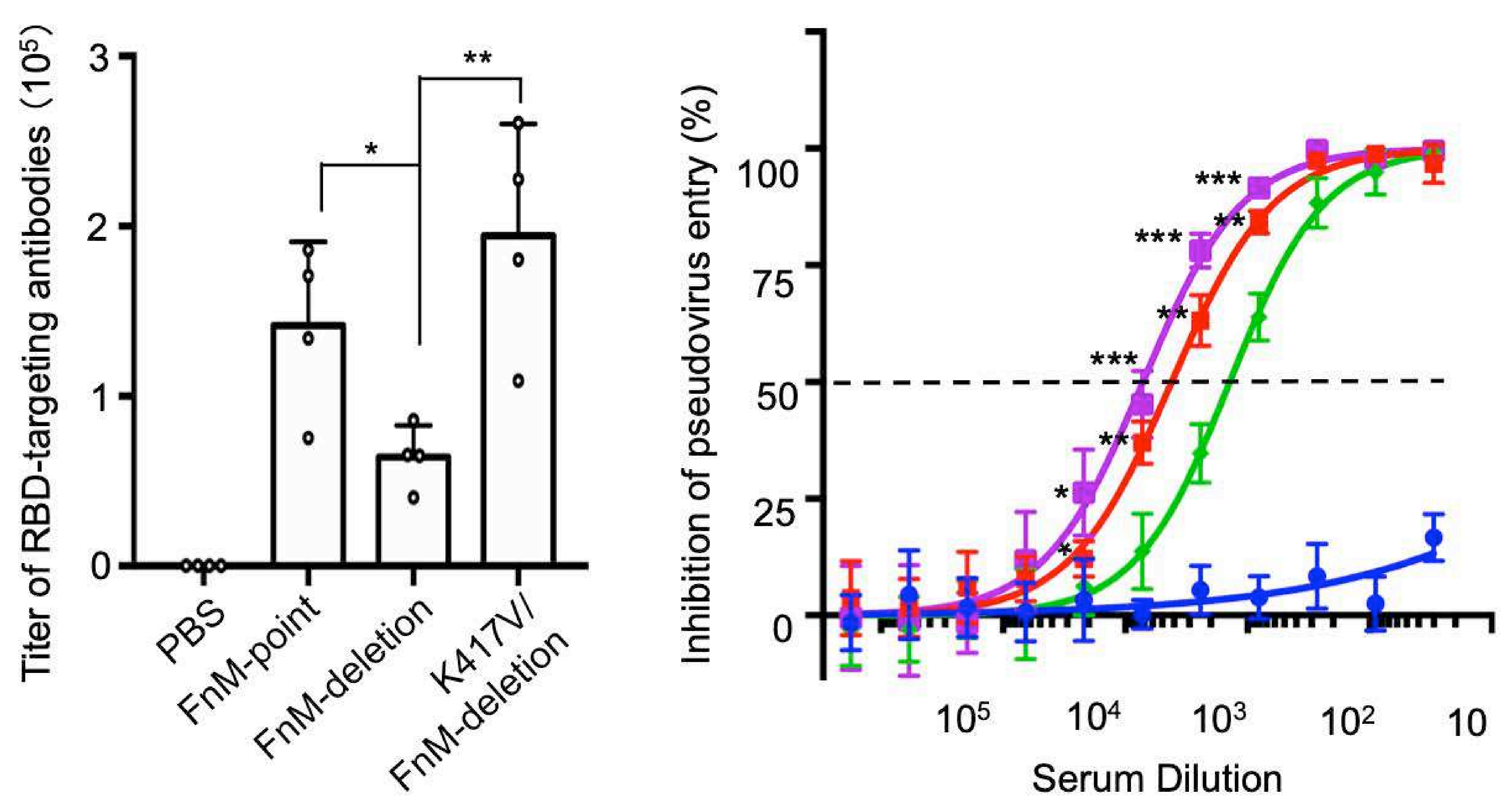

Sera from mice immunized by

$\mathrm{NT}_{50}$

(dilution factor)

$\sim$ PBS

FnM-point

4177

- FnM-deletion

1398

K417VI

FnM-deletion

7179

Figure 5 


\section{Table S1. Summary of previously determined cryo-EM structures of coronavirus spikes}

\begin{tabular}{|l|l|l|}
\hline Coronavirus & $\begin{array}{l}\text { RBD conformation of } \\
\text { spike protein }\end{array}$ & Reference \\
\hline Human SARS-CoV-2 & $\sim 50 \%$ open; $\sim 50 \%$ closed & (Walls et al., 2020) \\
\hline Bat RaTG13-CoV & Closed & (Wrobel et al., 2020) \\
\hline Pangolin SARS2-like CoV & Closed & (Wrobel et al., 2021) \\
\hline SARS-CoV-1 & Open & (Gui et al., 2017) \\
\hline MERS-CoV & Open & (Yuan et al., 2017) \\
\hline NL63-CoV & Closed & (Walls et al., 2016b) \\
\hline PEDV & Closed & (Wrapp and McLellan, 2019) \\
\hline 229E & Closed & (Li et al., 2019) \\
\hline FIPV & Closed & (Yang et al., 2020) \\
\hline MHV & Closed & (Walls et al., 2016a) \\
\hline IBV & Closed & (Shang et al., 2018a) \\
\hline PdCoV & Closed & (Shang et al., 2018b) \\
\hline HKU2 & Closed & (Yu et al., 2020) \\
\hline SADS & Closed & (Yu et al., 2020) \\
\hline & & \\
\hline
\end{tabular}

\section{References:}

Gui, M., Song, W., Zhou, H., Xu, J., Chen, S., Xiang, Y., and Wang, X. (2017). Cryoelectron microscopy structures of the SARS-CoV spike glycoprotein reveal a prerequisite conformational state for receptor binding. Cell Res 27, 119-129.

Li, Z., Tomlinson, A.C., Wong, A.H., Zhou, D., Desforges, M., Talbot, P.J., Benlekbir, S., Rubinstein, J.L., and Rini, J.M. (2019). The human coronavirus HCoV-229E S-protein structure and receptor binding. eLife 8 .

Shang, J., Zheng, Y., Yang, Y., Liu, C., Geng, Q., Luo, C., Zhang, W., and Li, F. (2018a). Cryo-EM structure of infectious bronchitis coronavirus spike protein reveals structural and functional evolution of coronavirus spike proteins. PLoS Pathog 14, e1007009.

Shang, J., Zheng, Y., Yang, Y., Liu, C., Geng, Q., Tai, W., Du, L., Zhou, Y., Zhang, W., and Li, F. (2018b). Cryo-Electron Microscopy Structure of Porcine Deltacoronavirus Spike Protein in the Prefusion State. J Virol 92.

Walls, A.C., Park, Y.J., Tortorici, M.A., Wall, A., McGuire, A.T., and Veesler, D. (2020). Structure, Function, and Antigenicity of the SARS-CoV-2 Spike Glycoprotein. Cell. 
Walls, A.C., Tortorici, M.A., Bosch, B.J., Frenz, B., Rottier, P.J., DiMaio, F., Rey, F.A., and Veesler, D. (2016a). Cryo-electron microscopy structure of a coronavirus spike glycoprotein trimer. Nature 531, 114-117.

Walls, A.C., Tortorici, M.A., Frenz, B., Snijder, J., Li, W., Rey, F.A., DiMaio, F., Bosch, B.J., and Veesler, D. (2016b). Glycan shield and epitope masking of a coronavirus spike protein observed by cryo-electron microscopy. Nat Struct Mol Biol 23, 899-905.

Wrapp, D., and McLellan, J.S. (2019). The 3.1-Angstrom Cryo-electron Microscopy Structure of the Porcine Epidemic Diarrhea Virus Spike Protein in the Prefusion Conformation. J Virol 93.

Wrobel, A.G., Benton, D.J., Xu, P., Calder, L.J., Borg, A., Roustan, C., Martin, S.R., Rosenthal, P.B., Skehel, J.J., and Gamblin, S.J. (2021). Structure and binding properties of Pangolin-CoV spike glycoprotein inform the evolution of SARS-CoV-2. Nature communications 12, 837.

Wrobel, A.G., Benton, D.J., Xu, P., Roustan, C., Martin, S.R., Rosenthal, P.B., Skehel, J.J., and Gamblin, S.J. (2020). SARS-CoV-2 and bat RaTG13 spike glycoprotein structures inform on virus evolution and furin-cleavage effects. Nat Struct Mol Biol 27, 763767.

Yang, T.J., Chang, Y.C., Ko, T.P., Draczkowski, P., Chien, Y.C., Chang, Y.C., Wu, K.P., Khoo, K.H., Chang, H.W., and Hsu, S.D. (2020). Cryo-EM analysis of a feline coronavirus spike protein reveals a unique structure and camouflaging glycans. Proc Natl Acad Sci U S A 117, 1438-1446.

Yu, J., Qiao, S., Guo, R., and Wang, X. (2020). Cryo-EM structures of HKU2 and SADS$\mathrm{CoV}$ spike glycoproteins provide insights into coronavirus evolution. Nature communications 11,3070 .

Yuan, Y., Cao, D., Zhang, Y., Ma, J., Qi, J., Wang, Q., Lu, G., Wu, Y., Yan, J., Shi, Y., et al. (2017). Cryo-EM structures of MERS-CoV and SARS-CoV spike glycoproteins reveal the dynamic receptor binding domains. Nature communications 8, 15092. 
Table S2. Cryo-EM data collection and model validation statistics

SARS-CoV-2 spike ectodomain (fusion motif deletion)

\begin{tabular}{ll}
\hline Data Collection & \\
\hline Microscope & Titan Krios \\
Voltage $(\mathrm{kV})$ & 300 \\
Camera & K2 summit \\
Camera model & $\begin{array}{l}\text { Super- } \\
\text { resolution }\end{array}$ \\
& $-0.6 \sim-2.6$ \\
Defocus range $(\mu \mathrm{m})$ & 8 \\
Exposure time $(\mathrm{s})$ & 4784 \\
Movies & 40 \\
Frames per movie & 1.564 \\
Dose rate $\left(e^{-} / \AA^{2} / \mathrm{s}\right)$ & 0.521 \\
Magnified pixel size $(\AA)$ & \\
\hline Reconstruction & $\mathrm{RELION} 3.0$ \\
\hline Software & $\mathrm{C} 3$ \\
Symmetry & 65,774 \\
Particles refined & 3.80 \\
Map Resolution $(\AA)$ & -166 \\
Map sharpening $B$-factor & \\
$\left(\AA^{2}\right)$ & \\
\hline Model Validation & \\
\hline MolProbity Score & 1.64 \\
All-atom clashscore & 5.54 \\
$\mathrm{C}_{\beta}$ deviations & 0 \\
Rotamer outliers $(\%)$ & 0 \\
Ramachandran & \\
Favored $(\%)$ & 95.08 \\
Allowed $(\%)$ & 4.92 \\
$\quad$ Outliers $(\%)$ & 0.00 \\
RMS deviations & \\
Bond length $(\AA)$ & 0.008 \\
Bond angles $\left({ }^{\circ}\right)$ & 0.818 \\
\hline
\end{tabular}

SARS2-CoV-2 spike ectodomain (fusion motif point mutations)

\begin{tabular}{ll}
\hline Data Collection & \\
\hline Microscope & Titan Krios \\
Voltage $(\mathrm{kV})$ & 300 \\
Camera & K2 summit \\
Camera model & $\begin{array}{l}\text { Super- } \\
\text { resolution }\end{array}$ \\
& $-0.6 \sim-2.6$ \\
Defocus range $(\mu \mathrm{m})$ & 8 \\
Exposure time $(\mathrm{s})$ & 1847 \\
Movies & 40 \\
Frames per movie & 1.564 \\
Dose rate $\left(e^{-} / \AA^{2} / \mathrm{s}\right)$ & 0.521 \\
Magnified pixel size $(\AA)$ & \\
\hline Reconstruction & RELION 3.0 \\
\hline Software & $\mathrm{C} 3$ \\
Symmetry & 28,863 \\
Particles refined & 4.37 \\
Map Resolution $(\AA)$ & -181 \\
Map sharpening $B$-factor & \\
$\left(\AA^{2}\right)$ & \\
\hline Model Validation & \\
\hline MolProbity Score & 1.70 \\
All-atom clashscore & 5.34 \\
$\mathrm{C}_{\beta}$ deviations & 0 \\
Rotamer outliers (\%) & 0.47 \\
Ramachandran & \\
$\quad$ Favored $(\%)$ & 93.88 \\
Allowed $(\%)$ & 6.12 \\
Outliers $(\%)$ & 0.00 \\
RMS deviations & \\
Bond length $(\AA)$ & 0.008 \\
Bond angles $\left({ }^{\circ}\right)$ & 0.866 \\
\hline &
\end{tabular}


Furin motif deletion

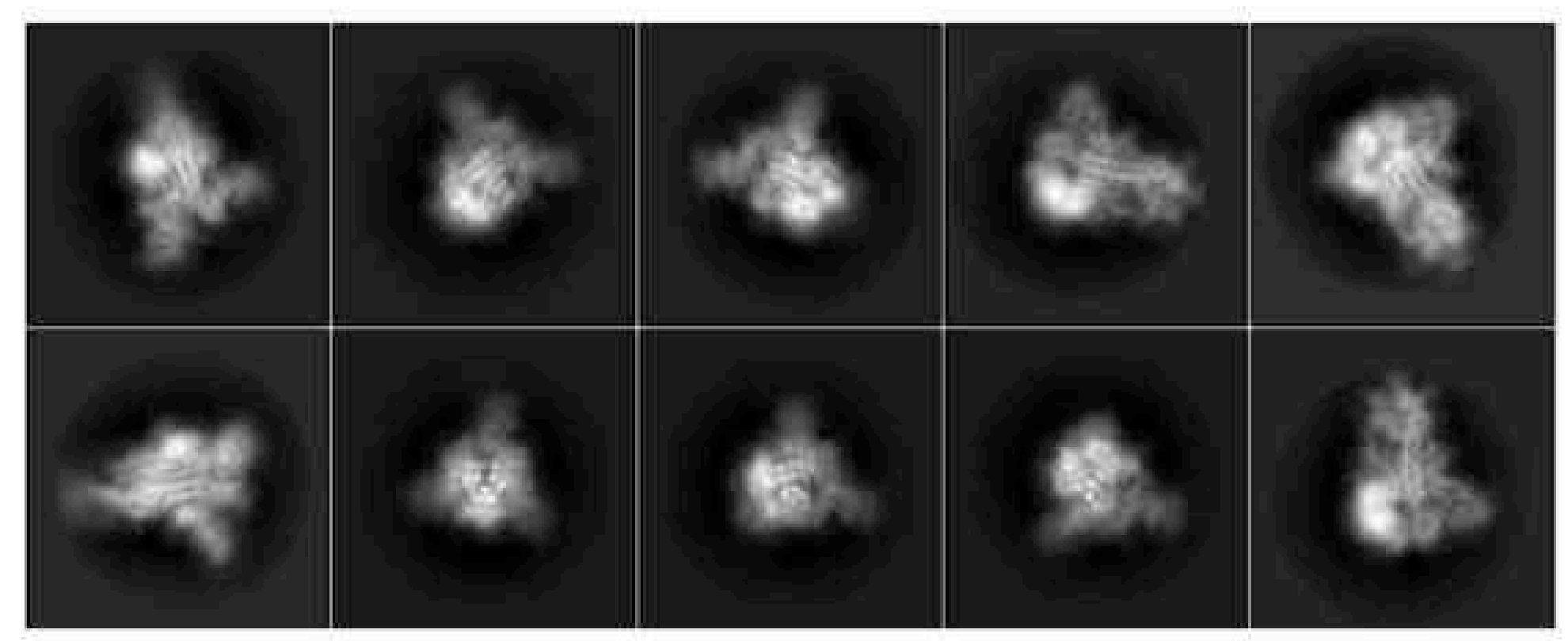

Furin motif point mutations

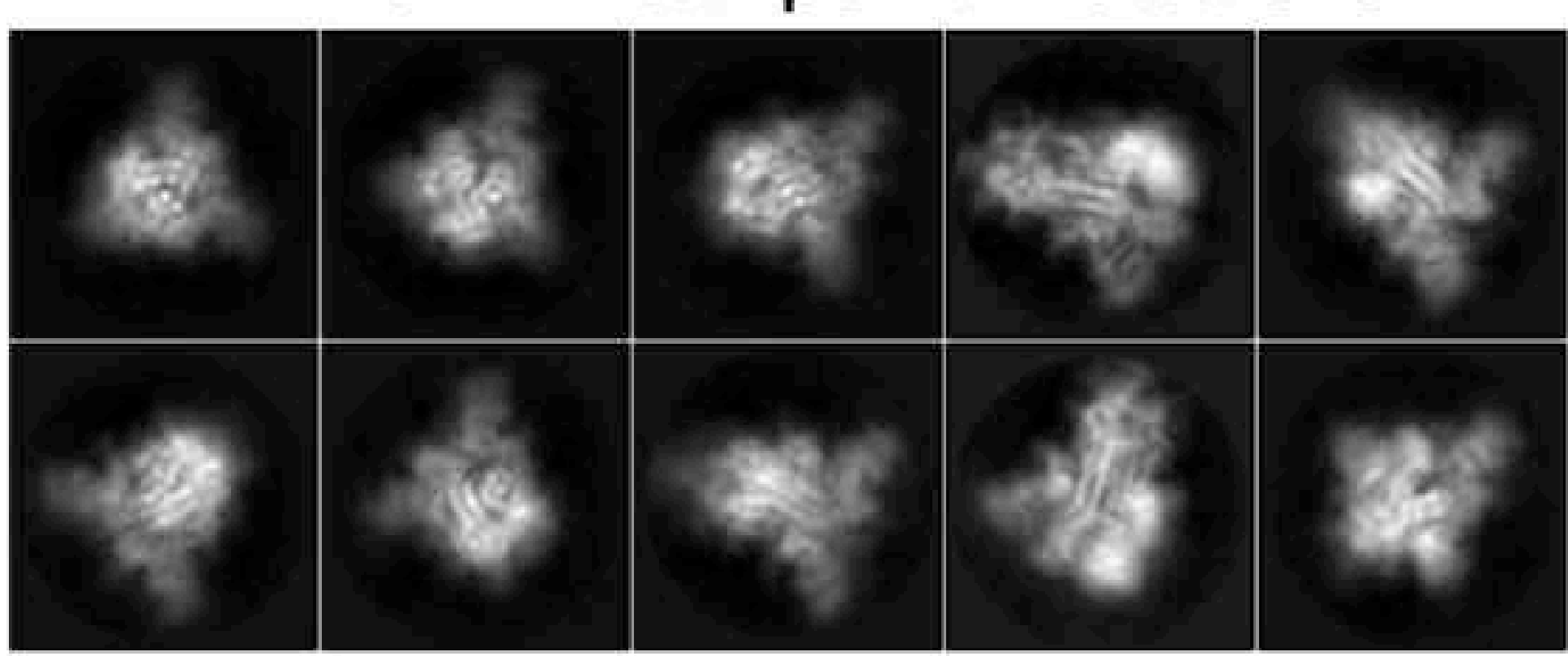

RBD mutation (K417V)

+ Furin motif deletion

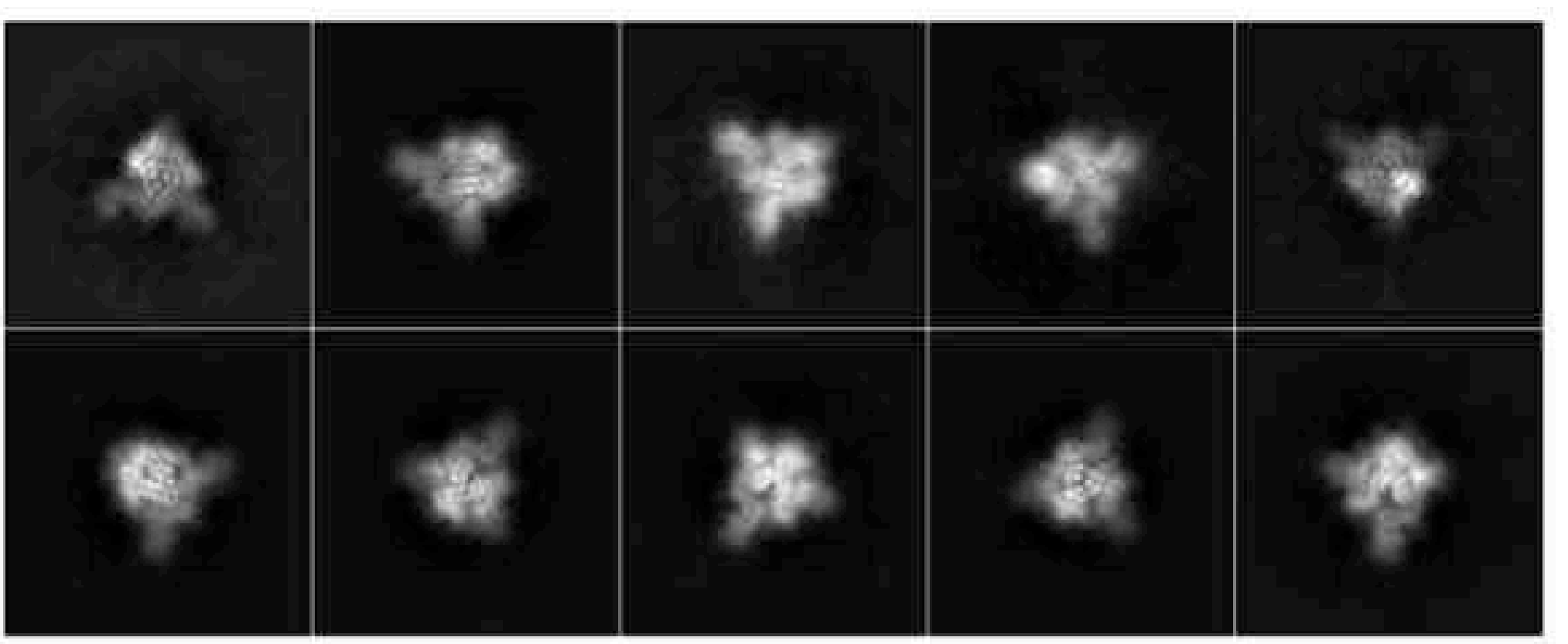

B

\begin{tabular}{|c|c|c|c|c|}
\hline & 3D classification & symmetry & $\begin{array}{l}\text { Final } \\
\text { number of } \\
\text { particles }\end{array}$ & $\begin{array}{l}\text { Final } \\
\text { resolution } \\
\text { of density }\end{array}$ \\
\hline $\begin{array}{l}\text { Furin motif } \\
\text { deletion }\end{array}$ & $\begin{array}{l}\text { Closed } \\
\text { (all RBDs down) }\end{array}$ & C3 & 65302 & $3.8 \AA$ \\
\hline \multirow{2}{*}{$\begin{array}{l}\text { Furin motif } \\
\text { point } \\
\text { mutations }\end{array}$} & $\begin{array}{l}\text { Closed } \\
\text { (all RBDs down) }\end{array}$ & C3 & 23849 & $4.4 \AA$ \\
\hline & $\begin{array}{l}\text { Open } \\
\text { (one RBD up; } \\
\text { two RBDs down) }\end{array}$ & C1 & 21894 & $5.3 \AA$ \\
\hline \multirow{2}{*}{$\begin{array}{l}\text { RBD } \\
\text { mutation } \\
\text { (K417V) } \\
+ \text { Furin } \\
\text { motif } \\
\text { deletion }\end{array}$} & $\begin{array}{l}\text { Closed } \\
\text { (all RBDs down) }\end{array}$ & C3 & 9502 & $4.6 \AA$ \\
\hline & $\begin{array}{l}\text { Open } \\
\text { (one RBD up; } \\
\text { two RBDs down) }\end{array}$ & C1 & 101413 & $4.6 \AA$ \\
\hline
\end{tabular}

Figure S1 


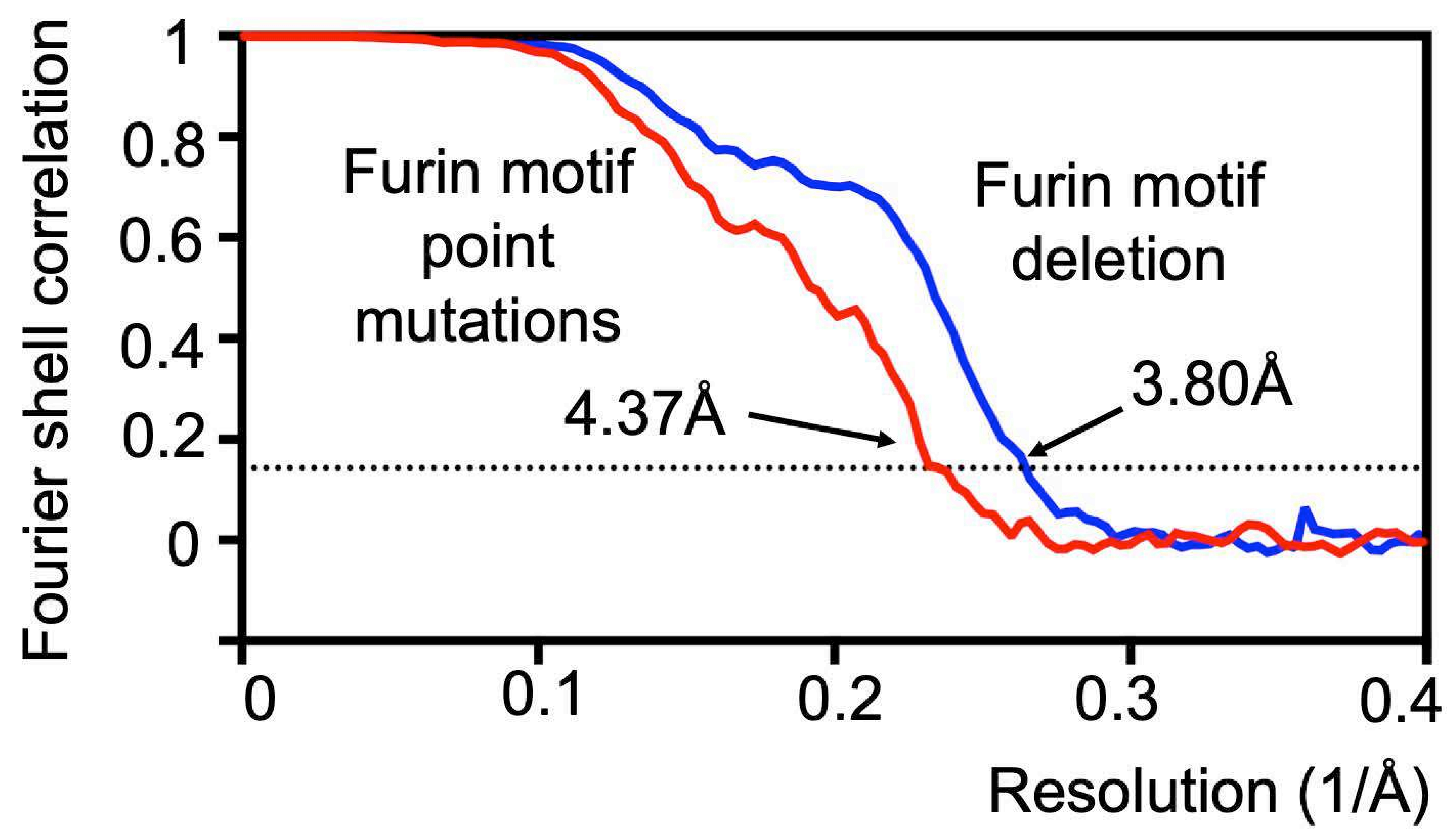

Figure S2

Furin motif deletion

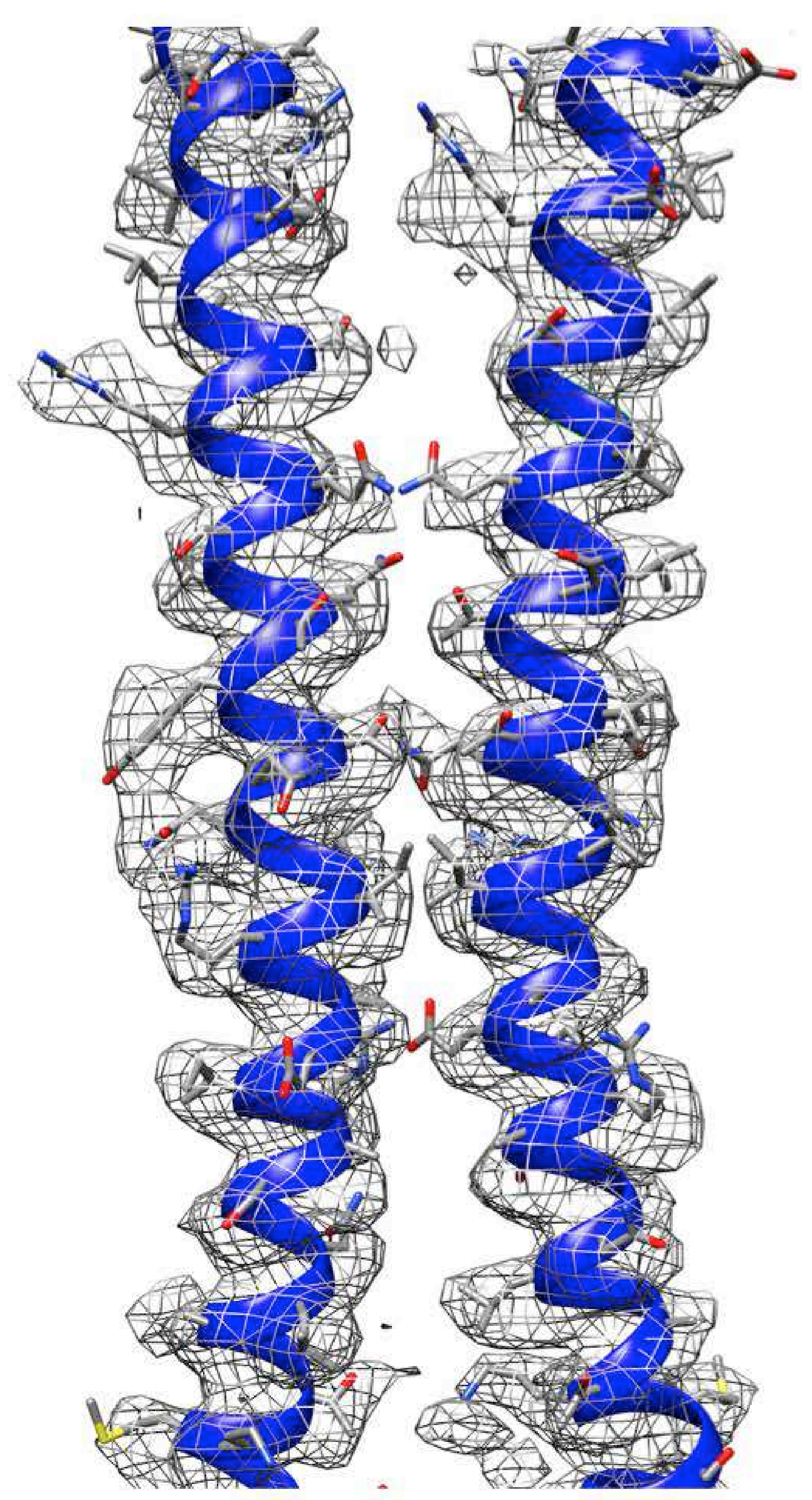

Furin motif point mutations

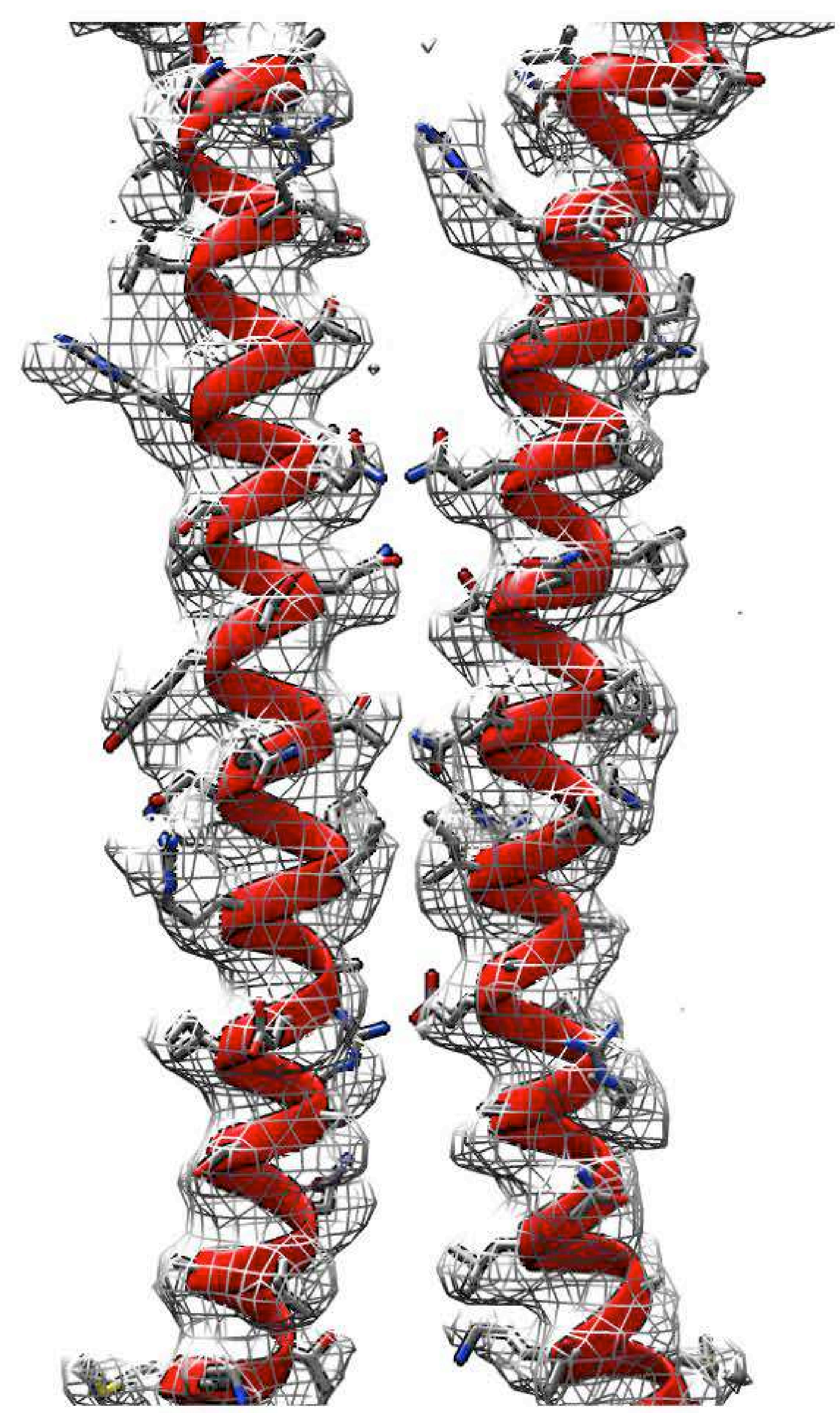


A

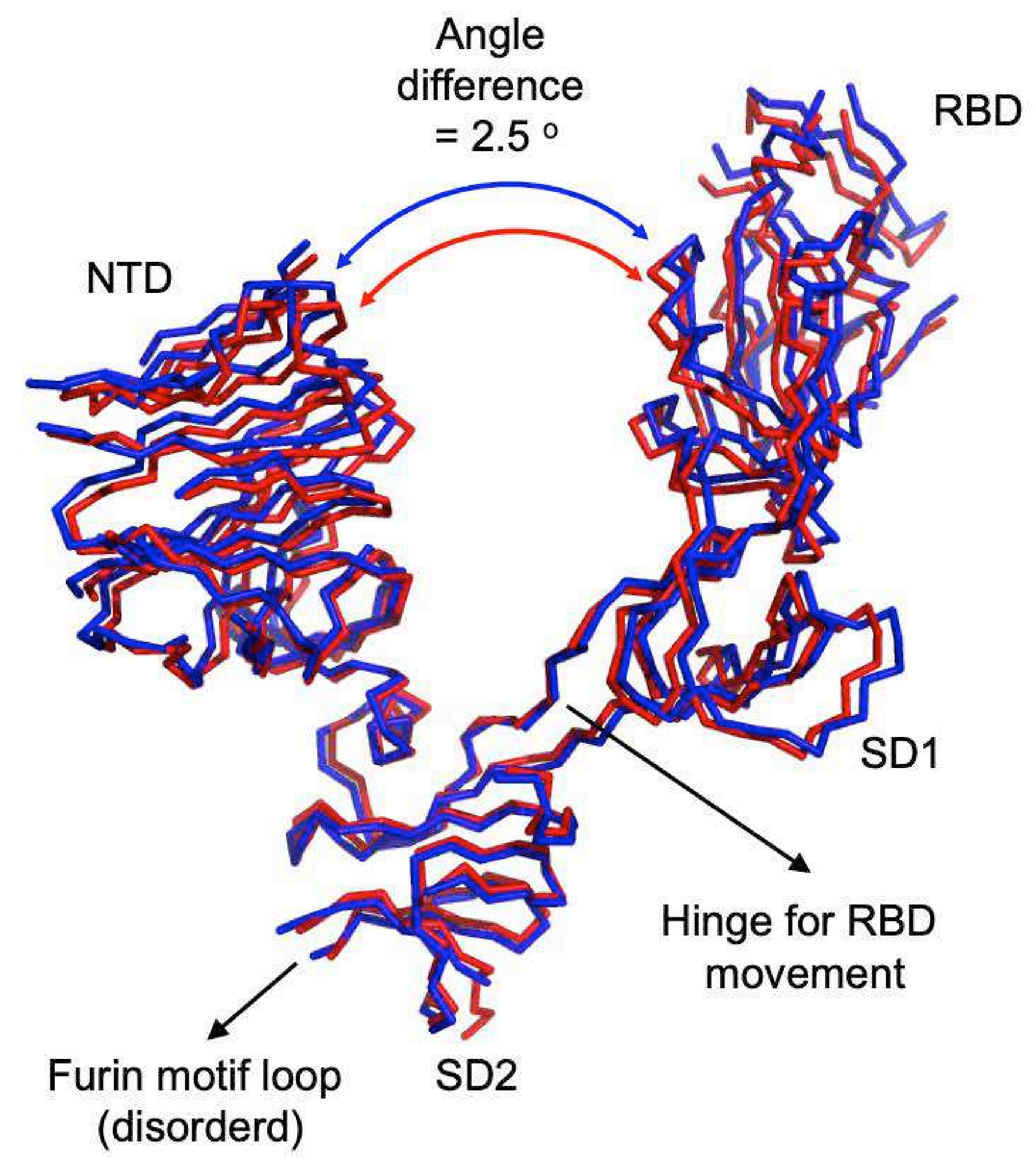

SARS-CoV-2 S1 monomer (furin motif point mutation)
SARS-CoV-2 S1 monomer (furin motif deletion)
B

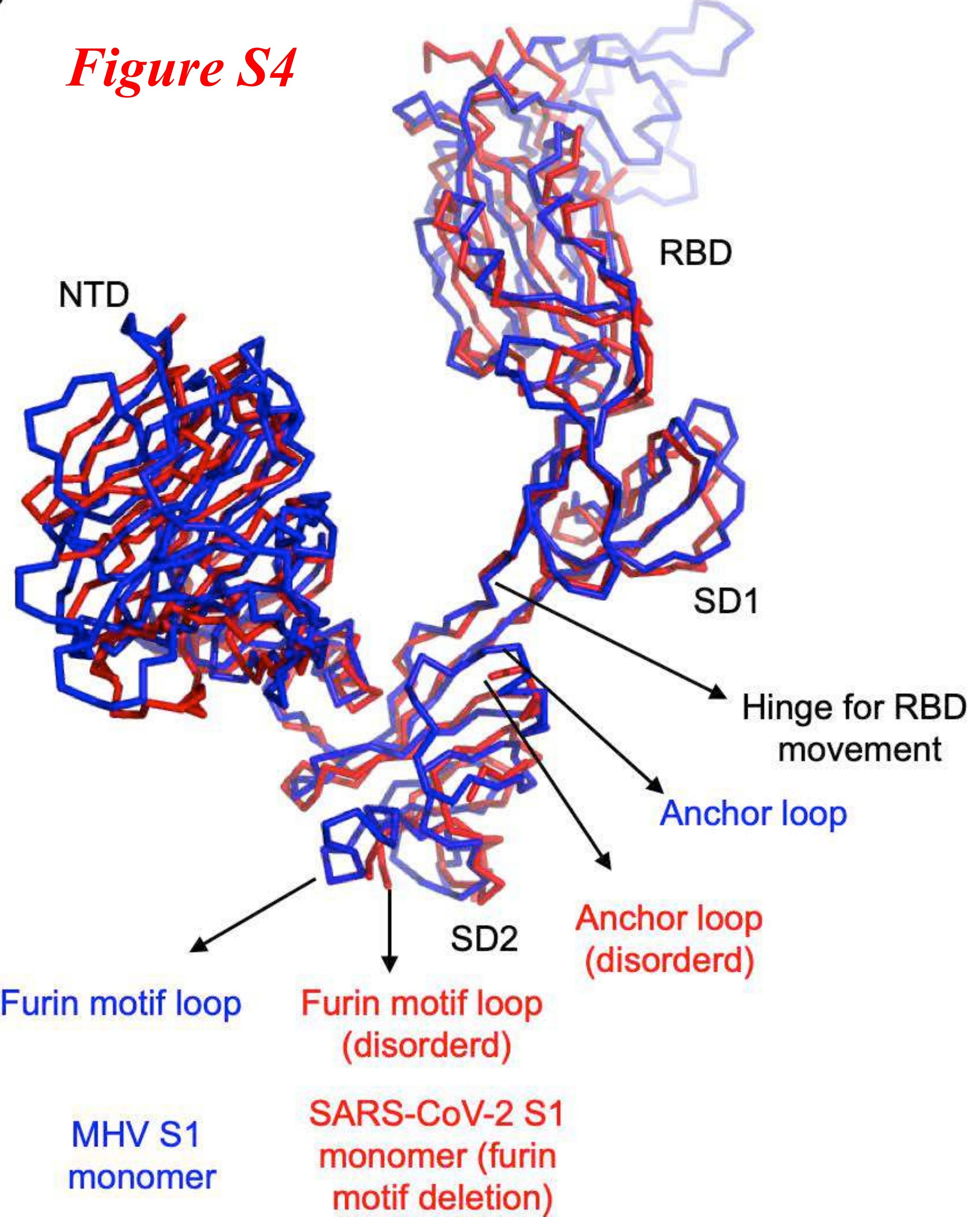

